\title{
Planar solutions of higher-spin theory. Nonlinear corrections
}

\section{V.E. Didenko and A.V. Korybut}

I.E. Tamm Department of Theoretical Physics, Lebedev Physical Institute, Leninsky prospect 53, 119991, Moscow, Russia

E-mail: didenko@lpi.ru, akoribut@gmail.com

ABSTRACT: Leading order higher-spin corrections to the linearized higher-spin black brane are analyzed in four dimensions. It is shown that the static solution that respects planar symmetry exists in the bosonic case at given order. Its higher-spin Weyl tensors are found in a closed form and are shown to have the double copy origin. The effect of higher-spin fields to form a strictly positive scalar condensate for any values of higher-spin charges is observed.

KeYwords: Higher Spin Gravity, Higher Spin Symmetry

ArXIV EPRINT: 2110.02256

In memory of Michael Soloviev 


\section{Contents}

1 Introduction 1

2 Higher-spin equations $\quad 4$

3 Current ansatz $\quad 8$

3.1 Global symmetry parameter and a flat connection 8

3.2 $\nabla$-covariant form of HS equations and the current ansatz $\quad 10$

4 Solutions $\quad 12$

$\begin{array}{lll}4.1 & \text { Linear solutions } & 12\end{array}$

$\begin{array}{lll}4.2 & \text { Quadratic analysis } & 14\end{array}$

$\begin{array}{lll}\text { 4.2.1 Static condition } & 15\end{array}$

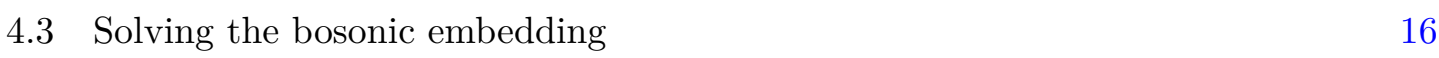

$\begin{array}{lll}\text { 4.3.1 Determining } \mathbf{c}(z) & 17\end{array}$

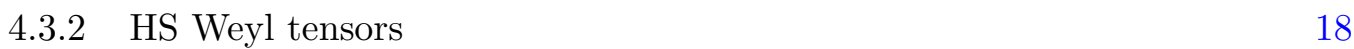

4.4 Properties of the quadratic corrections 20

5 Concluding remarks $\quad 22$

$\begin{array}{ll}\text { A Verification of consistency condition } & 24\end{array}$

B Explicit expression for $h(w, \bar{w}) \quad 26$

\section{Introduction}

This paper is a continuation of the $A d S / C F T$ [1-3] inspired study on higher-spin (HS) analogs of GR black branes. For a detailed motivation, extended introduction and references we refer to its first part [4], where a linearized step in this direction has been made and a closed form solution for the free HS gauge fields proposed. The natural question we address here is whether the HS brane like solution extends beyond free level and if yes how does it look like? To this end we consider HS equations of motion up to quadratic order.

The potential physical importance of this solution rests on the experience in $A d S / C F T$, where black branes of (super)gravity play a fundamental role as bulk dual states of thermal CFTs [5]. The dictionary is achieved via an AdS factor in the near horizon limit of the metric provided its Euclidian time points are properly identified to make it smooth, thus introducing a thermal $S^{1}$. In HS theory the line element associated with $s=2$ metric is no longer gauge invariant object. This seemingly revokes the above argument. However, since the notion of HS global symmetries as transformations that leave a particular solution invariant is similar to those in GR, it is tempting to speculate that solutions with planar symmetry of HS theory might be as important as they are in gravity from $A d S / C F T$ point of view. 
Our main findings are the following. By analyzing the deformation problem for planar solutions by interactions we come across with a certain integrability condition that turns out to be satisfied for the bosonic HS $A$-model. This allows us to find closed form expressions for HS Weyl tensors. These reveal the double copy structure and can be reconstructed in terms of multiple copies of Maxwell tensor and its scalar zeroth copy. A particular attention is paid to the linearized pure $s=2$ case corresponding to the standard GR black brane. While the linearized solution survives in the full GR, this is no longer so for HS interactions as spin two sector receives corrections. Moreover, HS interactions induce non zero $s=0$ and $s=4$ fields at leading order as well. We have also studied the effect of HS fields to the scalar sector of the theory and observed that they tend to induce a strictly positive scalar condensate irrespectively of the signs of HS charges.

Interacting HS framework is well elaborated at the level of the unfolded equations in four dimensions (see e.g., [6]). A schematic form of these equations is

$$
\begin{aligned}
\mathrm{d} \mathbf{w}+\mathbf{w} * \mathbf{w} & =\Upsilon(\mathbf{w}, \mathbf{w}, C)+\Upsilon(\mathbf{w}, \mathbf{w}, C, C)+\ldots, \\
\mathrm{d} C+[\mathbf{w}, C]_{*} & =\Upsilon(\mathbf{w}, C, C)+\Upsilon(\mathbf{w}, C, C, C)+\ldots,
\end{aligned}
$$

where $\mathbf{w}$ is the 1-form that encodes HS potentials, while $C$ is the 0-form that contains information about HS Weyl tensors. Fields $\mathbf{w}$ and $C$ both may appear in several copies like in e.g., supersymmetric models. $\Upsilon$ collectively denotes interaction vertices. Formally, these can be extracted up to field redefinition order by order from the generating Vasiliev system [7] which guarantees HS gauge invariance of vertices. However, the gauge invariance alone is not sufficient for having well-defined observables such as e.g., boundary correlation functions. This further restricts functional class of the Vasiliev master fields leading to the conjecture of spin locality $[8,9]$, which if true guarantees finiteness of classical theory. Whether spin locality applies to all orders is an open problem. Let us stress in this respect that there are alternative approaches to HS problem in the literature [10], where the locality is sacrificed already at cubic order, while the guiding principle is the bi-local approach of [11] that by construction gives proper boundary correlators at any order. The emergent space-time and HS fields dual to a thermal CFT was also studied in [12] in bi-local terms.

There are few exact solutions of the Vasiliev equations found over the years [13-23]. We however would like to analyze HS equations that follow from the generating system in perturbations rather than the Vasiliev system itself in this paper as this gives us control over locality of HS interactions at lowest orders which otherwise is hard to trace back. The detailed structure of these equations at leading order will be given below. For now we note that the manifest form of $\Upsilon$-vertices consistent with the locality criterium is highly nontrivial and by now is known to few orders only. Moreover, higher order locality itself seems so far problematic [24, 25] being a subject of active research [26-31].

Of our interest are leading order interactions in the sector of HS Weyl tensors (1.2) terminated by local $\Upsilon(\mathbf{w}, C, C)$. The corresponding perturbative expansion that we are going to work out is the following

$$
\begin{aligned}
& \mathbf{w}=\mathbf{w}^{(0)}+\mathbf{w}^{(1)}, \\
& C=C^{(1)}+C^{(2)},
\end{aligned}
$$


where $\mathbf{w}^{(0)}$ stands for the $A d S_{4}$ vacuum, while the linearized contribution $\mathbf{w}^{(1)}$ and $C^{(1)}$ have been found in [4]. So, the equation we examine here is

$$
\mathrm{d} C^{(2)}+\left[\mathbf{w}^{(0)}, C^{(2)}\right]_{*}+\left[\mathbf{w}^{(1)}, C^{(1)}\right]_{*}=\Upsilon\left(\mathbf{w}^{(0)}, C^{(1)}, C^{(1)}\right) .
$$

Its static, planar solution for $C^{(2)}$, if exists, contains second order corrections to HS black brane like Weyl tensors that we would like to find and analyze.

The linearized solution [4] we are going to use for $\mathbf{w}^{(1)}$ and $C^{(1)}$ corresponds to HS planar excitations of all integer spins each having its own 'mass like' real parameter $m_{s}$. The reason we refer to it as to a HS analog of a black brane rests on its space-time symmetry and its classical double copy form rather than on any physical characteristics like the presence of a horizon, which is by far beyond the reach in HS theory. Nevertheless, many black hole solutions admit HS generalization by forming a multiple copy of a certain spin $s=1$ field

$$
\mathrm{Weyl}_{s} \sim(\text { Maxwell })^{s},
$$

which in four dimensions says that the (anti)selfdual part of the linearized spin $s$ Weyl tensor that appears on the left hand side of this correspondence is given by $s$ copies of (anti)self-dual Maxwell tensor. Relation (1.6) gets exact upon putting in a proper dependence on a massless scalar field $\phi$ instead of $\sim$, which itself turns out to be made of $s=1$ Maxwell tensor. The case $s=2$ reproduces this way Petrov $D$ type Weyl tensor that includes black hole solutions on AdS. In terms of gauge potentials the multiple copies are realized via the Kerr-Schild ansatz

$$
\phi_{\mu_{1} \ldots \mu_{s}}=\phi k_{\mu_{1}} \ldots k_{\mu_{s}}
$$

which satisfies massless spin $s$ equation on AdS, provided $k_{\mu}$ is the so-called Kerr-Schild vector. It makes Einstein equations linearly exact, in particular. Eq. (1.6) for $s=2$ is called the Weyl double copy while its higher-spin extension is naturally calling the Weyl multi-copy. Similarly, (1.7) is the Kerr-Schild double copy for $s=2$ with the multi-copy extension for any $s$. Properties (1.6) and (1.7) both were originally observed within the unfolded approach in [16, 32, 33] for all four dimensional AdS black holes (including black branes) and then for the AdS Kerr-Schild ones in five dimensions ${ }^{1}$ [34]. The classical double copy origin of Kerr-Schild black holes rests on their algebraically special form, namely the $D$ Petrov type and thus may not be directly related to the original KLT relations [37]. Nevertheless there seems to be a substantial recent progress in understanding the classical double copy beyond algebraically special solutions [38-47].

For our means we will greatly exploit the type $D$ structure of GR black holes and in particular the linearized HS black brane solution. It was shown that the Weyl double copy naturally results from a general AdS global symmetry parameter in [33] via the Penrose like transform $^{2}[4,16]$. Technically this allows us plugging that parameter in the HS equations

\footnotetext{
${ }^{1}$ In the double copy literature properties (1.7) and (1.6) for asymptotically Minkowski black holes $(s=2)$ were independently observed in $[35,36]$.

${ }^{2}$ From the twistor stand point this parameter literally corresponds to a rank two dual twistor on asymptotically Minkowski space, see [41, 43].
} 
of motion serving as a pillar for searching for their solutions with the required symmetry. This gives us on the one hand a covariant approach with respect to planar symmetry and on the other considerably simplifies the analysis. In doing so we observe that the parameter corresponding to the planar case induces a flat connection [4] that builds in into HS equations making planar symmetry manifest.

We find the so called current ansatz very useful for analysis of interactions. Originally introduced in [48] for establishing HS bulk-boundary correspondence between fields and currents at first order, it turns out to be applicable at second order too [49] leading to a non-linear equation for HS currents. Remarkably, the linearized planar solutions of [4] correspond to very simple conserved currents. Namely, a spin $s$ planar excitation is given by a power $s$ monomial current. This makes finding HS planar corrections in the current space natural. To this end we covariantize current ansatz of [48] using the AdS global symmetry parameter as a kind of a compensator field and analyze second order HS equation of motion in terms of currents.

We use the $4 d$ supersymmetric Vasiliev HS equations [6]. This gives us several options for embeddings of the linearized solution. Most of the time we study the usual bosonic embedding. Another natural option is the so called chiral embedding that appears upon association of mostly positive helicity part of the linearized solution with one of the two HS master zero-forms from the supersymmetric twisted-adjoint sector, while mostly negative with another one. Quite interestingly, the integrability condition that makes quadratic corrections a deformation of the linearized solution does not hold for chiral embedding. This means that either static planar solutions do not exist beyond free level in this case or they do not follow from deformation. In the latter case they can not support the double copy either.

The paper is organized as follows. In section 2 we briefly recall the structure of Vasiliev unfolded HS equations and give the explicit form of schematically written eq. (1.5). In section 3 HS equations are rewritten in terms of the so called current ansatz in a covariant with respect to planar symmetry way. In section 4 we find solution to those equations, provide details of its derivation and list important properties. Concluding remarks are collected in the last section. Paper is supplemented with two appendices one of which contains explicit check of the static consistency condition, while another one includes expression for function $h$ introduced in section 4.3.

\section{Higher-spin equations}

HS interactions (1.1), (1.2) can be extracted from the Vasiliev generating system [6]. The procedure is perturbative and starts with some exact solution.

Vacuum. The simplest HS vacuum state satisfies

$$
\begin{aligned}
\mathrm{d} \mathbf{w}+\mathbf{w} * \mathbf{w} & =0, \\
C & =0 .
\end{aligned}
$$

where $\mathbf{w}=\mathbf{w}(y, \bar{y} \mid x)$ is a 1 -form that depends on the auxiliary $\operatorname{sp}(4)$ spinor variable $Y_{A}=$ $\left(y_{\alpha}, \bar{y}_{\dot{\alpha}}\right)$. Decomposition of $\mathbf{w}$ in terms of $y$ 's describes HS gauge field sector. Non vacuum 
$C=C(y, \bar{y} \mid x)$ depends on $y$ 's too encoding gauge invariant physical degrees of freedom propagating on some vacuum state $\mathbf{w}^{(0)}$. Star-product $*$ is an associative product

$$
f(Y) * g(Y)=f(Y) e^{i \epsilon^{A B} \overleftarrow{\partial}_{A} \vec{\partial}_{B}} g(Y)
$$

that defines HS algebra as the enveloping one of the following oscillator relations

$$
\left[y_{\alpha}, y_{\beta}\right]_{*}=2 i \epsilon_{\alpha \beta}, \quad\left[y_{\alpha}, \bar{y}_{\dot{\beta}}\right]_{*}=0, \quad\left[\bar{y}_{\dot{\alpha}}, \bar{y}_{\dot{\beta}}\right]_{*}=2 i \epsilon_{\dot{\alpha} \dot{\beta}} .
$$

Purely gravitational solution of (2.1) describes $A d S_{4}$ and is given by

$$
\mathbf{w}^{(0)}=-\frac{i}{4}\left(\omega^{\alpha \beta} y_{\alpha} y_{\beta}+\bar{\omega}^{\dot{\alpha} \dot{\beta}} \bar{y}_{\dot{\alpha}} \bar{y}_{\dot{\beta}}+2 \mathbf{e}^{\alpha \dot{\beta}} y_{\alpha} \bar{y}_{\dot{\beta}}\right)
$$

where Lorentz connection $\omega^{\alpha \beta}, \bar{\omega}^{\dot{\alpha} \dot{\beta}}$ and vierbein $\mathbf{e}^{\alpha \dot{\beta}}$ satisfy Cartan structure equations ${ }^{3}$ of AdS

$$
\begin{aligned}
\mathrm{d} \omega_{\alpha \beta}-\omega_{\alpha}^{\gamma} \omega_{\gamma \beta}-\mathbf{e}_{\alpha} \dot{\gamma} \mathbf{e}_{\beta \dot{\gamma}} & =0, \\
\mathrm{~d} \mathbf{e}_{\alpha \dot{\alpha}}-\omega_{\alpha}{ }^{\beta} \mathbf{e}_{\beta \dot{\alpha}}-\bar{\omega}_{\dot{\alpha}} \dot{\beta} \mathbf{e}_{\alpha \dot{\beta}} & =0 .
\end{aligned}
$$

Free equations. Free propagation of HS fields around AdS vacuum (2.5) is described by the gauge invariant twisted-adjoint covariant constancy condition on the 0-form $\mathcal{C}(y, \bar{y} ; k, \bar{k} \mid x)$ and by the on-mass-shell condition in the sector of gauge fields parameterized by 1 -form $\mathbf{w}(y, \bar{y} ; k, \bar{k} \mid x)$. Here, $k$ and $\bar{k}$ are the two Clifford elements that make HS system $\mathcal{N}=2$ supersymmetric satisfy

$$
\begin{aligned}
& \left\{k, y_{\alpha}\right\}=0, \quad\left[k, \bar{y}_{\dot{\alpha}}\right]=0, \quad k^{2}=1, \\
& \left\{\bar{k}, \bar{y}_{\dot{\alpha}}\right\}=0, \quad\left[\bar{k}, y_{\alpha}\right]=0, \quad \bar{k}^{2}=1, \\
& {[k, \bar{k}]=0 .}
\end{aligned}
$$

Dynamical master fields are singled out by the following condition

$$
\mathbf{w}(y, \bar{y} ;-k,-\bar{k} \mid x)=\mathbf{w}(y, \bar{y} ; k, \bar{k} \mid x), \quad \mathcal{C}(y, \bar{y} ;-k,-\bar{k} \mid x)=-\mathcal{C}(y, \bar{y} ; k, \bar{k} \mid x) .
$$

Those that do not satisfy (2.11) are the so called topological fields [6], which describe no propagating degrees of freedom and we set them to zero in what follows meaning that

$$
\begin{aligned}
\mathcal{C} & =C(y, \bar{y} \mid x) k+\bar{C}(y, \bar{y} \mid x) \bar{k}, \\
\mathbf{w} & =\omega(y, \bar{y} \mid x)+\omega^{\prime}(y, \bar{y} \mid x) k \bar{k} .
\end{aligned}
$$

Proper reality conditions on master fields should be further imposed

$$
\mathbf{w}^{\dagger}=-\mathbf{w}, \quad \mathcal{C}^{\dagger}=\mathcal{C},
$$

where

$$
y_{\alpha}^{\dagger}=\bar{y}_{\dot{\alpha}}, \quad k^{\dagger}=\bar{k},
$$

\footnotetext{
${ }^{3}$ We set negative cosmological constant to a number for convenience.
} 
implying

$$
\begin{aligned}
C^{\dagger} & =\bar{C}(y,-\bar{y}), & \bar{C}^{\dagger} & =C(-y, \bar{y}), \\
\omega^{\dagger} & =-\omega(y, \bar{y}), & \omega^{\prime \dagger} & =-\omega^{\prime}(-y,-\bar{y}) .
\end{aligned}
$$

For the purely bosonic spin-statistics which is our case of interest one has in addition

$$
\mathcal{C}(y,-\bar{y})=\mathcal{C}(-y, \bar{y}), \quad w(y,-\bar{y})=w(-y, \bar{y}) .
$$

Free field dynamics is governed by the following equation of motion [50]

$$
D_{0} \mathbf{w}=\frac{i \bar{\eta}}{4} \mathbf{e}^{\alpha \dot{\gamma}} \mathbf{e}^{\beta} \dot{\gamma} \frac{\partial^{2}}{\partial y^{\alpha} \partial y^{\beta}} \mathcal{C}(y, 0 \mid x) \bar{k}+\frac{i \eta}{4} \mathbf{e}^{\gamma \dot{\alpha}} \mathbf{e}_{\gamma} \dot{\beta} \frac{\partial^{2}}{\partial \bar{y}^{\dot{\alpha}} \partial \bar{y}^{\dot{\beta}}} \mathcal{C}(0, \bar{y} \mid x) k,
$$

where

$$
D_{0}=\mathrm{d}+\omega^{\alpha \beta} y_{\alpha} \frac{\partial}{\partial y^{\beta}}+\bar{\omega}^{\dot{\alpha} \dot{\beta}} \bar{y}_{\dot{\alpha}} \frac{\partial}{\partial \bar{y}^{\dot{\beta}}}+\mathbf{e}^{\alpha \dot{\beta}}\left(y_{\alpha} \frac{\partial}{\partial \bar{y}^{\dot{\beta}}}+\bar{y}_{\dot{\beta}} \frac{\partial}{\partial y^{\alpha}}\right)
$$

is the $A d S_{4}$ covariant derivative and $\eta$ is an arbitrary constant phase factor which generally breaks parity of the theory beyond free level unless $\eta=1$ or $\eta=i$ (see [51]). In this paper we consider parity even case with $\eta=1$. Master field $\mathcal{C}$ is subject to the twisted-adjoint constancy condition

$$
D \mathcal{C}-i \mathbf{e}^{\alpha \dot{\alpha}}\left(y_{\alpha} \bar{y}_{\dot{\alpha}}-\frac{\partial}{\partial y^{\alpha}} \frac{\partial}{\partial \bar{y}^{\dot{\alpha}}}\right) \mathcal{C}=0
$$

where $D$ is the Lorentz covariant derivative

$$
D=\mathrm{d}+\omega^{\alpha \beta} y_{\alpha} \frac{\partial}{\partial y^{\beta}}+\bar{\omega}^{\dot{\alpha} \dot{\beta}} \bar{y} \dot{\alpha} \frac{\partial}{\partial \bar{y}^{\dot{\beta}}} .
$$

Quadratic corrections. Local HS non-linear corrections at leading order can be systematically extracted from Vasiliev equations [26]. The resulting contribution to the right hand side of (2.21) that contains the minimal number of derivatives acquires the following form ${ }^{4}$

$$
\begin{aligned}
D \mathcal{C} & -i \mathbf{e}^{\alpha \dot{\alpha}}\left(y_{\alpha} \bar{y}_{\dot{\alpha}}-\frac{\partial}{\partial y^{\alpha}} \frac{\partial}{\partial \bar{y}^{\dot{\alpha}}}\right) \mathcal{C} \\
= & -[\mathbf{w}, \mathcal{C}]_{*} \\
& -\frac{\eta}{2} \mathbf{e}^{\alpha \dot{\alpha}} y_{\alpha} \int \frac{\mathrm{d} \bar{u} \mathrm{~d} \bar{v}}{(2 \pi)^{2}} \int_{0}^{1} \mathrm{~d} \tau e^{i \bar{u}_{\dot{\alpha}} \bar{v}^{\dot{\alpha}}}\left((1-\tau) \partial_{1}-\tau \partial_{2}\right)_{\dot{\alpha}} \mathcal{C}(\tau y, \bar{y}+\bar{u}) \mathcal{C}(-(1-\tau) y, \bar{y}+\bar{v}) k \\
& -\frac{\bar{\eta}}{2} \mathbf{e}^{\alpha \dot{\alpha}} \bar{y}_{\dot{\alpha}} \int \frac{\mathrm{d} u \mathrm{~d} v}{(2 \pi)^{2}} \int_{0}^{1} \mathrm{~d} \tau e^{i u_{\alpha} v^{\alpha}}\left((1-\tau) \partial_{1}-\tau \partial_{2}\right)_{\alpha} \mathcal{C}(y+u, \tau \bar{y}) \mathcal{C}(y+v,(\tau-1) \bar{y}) \bar{k}
\end{aligned}
$$

where star-product $*$ is attributed to the HS algebra and realized via the Moyal product $(2.3)$ and $\partial_{1 \dot{\alpha}}, \partial_{1 \alpha}$ differentiate argument of the first field (as seen from the left) of the two $\mathcal{C C}$ in $(2.23)$, while $\partial_{2 \dot{\alpha}}, \partial_{2 \alpha}$ act on the second $\mathcal{C}$ correspondingly. One should be cautious with Kleinians $k$ and $\bar{k}$ when performing $\bar{u}, \bar{v}$-integration. General rule is to drag

\footnotetext{
${ }^{4}$ The vertex contains bosons and fermions as well as parity breaking parameter $\eta$. Its bosonic reduction for type- $A$ model with $\eta=1$ is known in the partially gauged fixed form at the Lagrangian level [53].
} 
all the Kleinians that may enter fields $\mathcal{C}^{\prime} s$ and $\mathbf{w}$, say, to the right using (2.8)-(2.10) before integration. ${ }^{5}$

There are two natural embeddings of the bosonic fields one of each spin into the $\mathcal{N}=2$ supersymmetric theory with two fields of each spin.

Bosonic embedding There is a well known truncation of the supersymmetric theory that leads to the bosonic HS model having each field appearing once. It is governed by certain automorphism of the full Vasiliev equations, which allows one to set

$$
\begin{aligned}
\mathcal{C} & =C(y, \bar{y} \mid x)(k+\bar{k}), & \mathbf{w} & =\omega(y, \bar{y} \mid x)(1+k \bar{k}) \\
C(y,-\bar{y}) & =C(-y, \bar{y}), & \omega(y,-\bar{y}) & =\omega(-y, \bar{y}),
\end{aligned}
$$

where master fields $C$ and $\omega$ describe single copy of each integer spin. Substituting (2.24) into (2.23) one arrives at the equation for quadratic HS correction for fields $s=0,1,2, \ldots$

$$
\begin{aligned}
D C & -i \mathbf{e}^{\alpha \dot{\alpha}}\left(y_{\alpha} \bar{y}_{\dot{\alpha}}-\frac{\partial}{\partial y^{\alpha}} \frac{\partial}{\partial \bar{y}^{\dot{\alpha}}}\right) C \\
= & -\omega * C+C * \pi(\omega) \\
& -\eta \mathbf{e}^{\alpha \dot{\alpha}} y_{\alpha} \int \frac{\mathrm{d} \bar{u} \mathrm{~d} \bar{v}}{(2 \pi)^{2}} \int_{0}^{1} \mathrm{~d} \tau e^{i \bar{u}_{\dot{\alpha}} \bar{v}^{\dot{\alpha}}}\left((1-\tau) \partial_{1}-\tau \partial_{2}\right)_{\dot{\alpha}} C(\tau y, \bar{y}+\bar{u}) C((1-\tau) y, \bar{y}+\bar{v}) \\
& -\bar{\eta} \mathbf{e}^{\alpha \dot{\alpha}} \bar{y}_{\dot{\alpha}} \int \frac{\mathrm{d} u \mathrm{~d} v}{(2 \pi)^{2}} \int_{0}^{1} \mathrm{~d} \tau e^{i u_{\alpha} v^{\alpha}}\left((1-\tau) \partial_{1}-\tau \partial_{2}\right)_{\alpha} C(y+u, \tau \bar{y}) C(y+v,(1-\tau) \bar{y}
\end{aligned}
$$

where

$$
\pi f(y, \bar{y})=f(-y, \bar{y}), \quad \bar{\pi} f(y, \bar{y})=f(y,-\bar{y}) .
$$

For bosonic fields $\pi f=\bar{\pi} f$.

Chiral embedding. While the bosonic embedding is defined at the level of full equations of motion, there is yet another embedding capturing each spin $s$ field only once that can be defined at least perturbatively in four dimensions. Indeed, consider (2.12). Since $C$ is complex it corresponds to two real fields in general. We can however set in (2.12)

$$
C(y, \bar{y} \mid x)=C^{+}(y, \bar{y} \mid x), \quad \bar{C}(y, \bar{y} \mid x)=C^{-}(y, \bar{y} \mid x),
$$

where $C^{+}$is the helicity positive part and $C^{-}$is the helicity negative one of one and the same field $C^{+}+C^{-}$describing a real HS module. Since both $C^{ \pm}$satisfy $(2.21)$ and

$$
\bar{C}^{ \pm}=C^{\mp}
$$

it follows that (2.12) and (2.13)

$$
\mathcal{C}=C^{+} k+C^{-} \bar{k}, \quad \mathbf{w}=\omega(1+k \bar{k})
$$

describe each $\operatorname{spin} s$ once, where we take for $s>0$

$$
C^{-}(0, \bar{y})=0, \quad C^{+}(y, 0)=0 .
$$

\footnotetext{
${ }^{5}$ Note that in doing so the (anti)holomorphic Klein operator flips the sign of the whole (anti)holomorphic argument of $\mathcal{C}$ or $w$ rather than $(\bar{y}) y$ alone.
} 


\section{Current ansatz}

We now proceed with the so called current form of HS equations introduced in [48] at free level within the $A d S / C F T$ approach. It is designed to extract HS current module from fields in the twisted-adjoint. By construction it uses $3+1$ decomposition in terms of boundary coordinates $x^{i}$ and radial $z$. We want to do something similar but adjusted to planar symmetry. We prefer to stay covariant however and for that reason introduce a proper $A d S_{4}$ global symmetry parameter following [4].

\subsection{Global symmetry parameter and a flat connection}

Planar solutions of the linear HS equations both in the twisted-adjoint (2.21) and adjoint (2.19) sectors have been recently obtained using the generating AdS global symmetry parameter in [4]. The planar symmetry also provides one with an auxiliary flat connection which plays a crucial role in solving gauge field sector. All necessary details of that construction can be found in [4]. Here we briefly recall the necessary formulae, which we are going to use in what follows.

The $A d S_{4}$ global symmetry parameter $K_{A B}=K_{B A}$

$$
K_{A B}=\left(\begin{array}{cc}
\varkappa_{\alpha \beta} & v_{\alpha \dot{\beta}} \\
v_{\beta \dot{\alpha}} & \bar{\varkappa}_{\dot{\alpha} \dot{\beta}}
\end{array}\right), \quad \varkappa_{\alpha \beta}=\varkappa_{\beta \alpha}, \quad \bar{\varkappa}_{\dot{\alpha} \dot{\beta}}=\bar{\varkappa}_{\dot{\beta} \dot{\alpha}}
$$

is known to generate $D$-type solutions ${ }^{6}$ both in GR [33] and in HS theory [16]. It satisfies

$$
\begin{aligned}
D \varkappa_{\alpha \beta} & =\mathbf{e}_{\alpha}{ }^{\dot{\gamma}} v_{\beta \dot{\gamma}}+\mathbf{e}_{\beta}^{\dot{\gamma}} v_{\alpha \dot{\gamma}}, \\
D v_{\alpha \dot{\alpha}} & =\mathbf{e}_{\alpha} \dot{\gamma} \bar{\varkappa}_{\dot{\alpha} \dot{\gamma}}+\mathbf{e}^{\gamma} \dot{\alpha} \varkappa_{\gamma \alpha},
\end{aligned}
$$

where $v_{\alpha \dot{\beta}}$ is an $A d S_{4}$ Killing vector, while $\varkappa_{\alpha \beta}, \bar{\varkappa}_{\dot{\alpha} \dot{\beta}}$ are the (anti)self-dual components of the so called closed conformal Killing-Yano tensor (see [4] for more details). While different $K$ 's correspond to different type of solutions it generates, the planar case is singled out by the following condition ${ }^{7}$

$$
K_{A}^{C} K_{C B}=0, \quad z^{-2}:=-\frac{1}{2} \varkappa_{\alpha \beta} \varkappa^{\alpha \beta}=-\frac{1}{2} \bar{\varkappa}_{\dot{\alpha} \dot{\beta}} \varkappa^{\alpha \beta} \geq 0
$$

or more explicitly

$$
\begin{aligned}
\varkappa_{\alpha}^{\gamma} \varkappa_{\gamma \beta}+v_{\alpha}^{\dot{\gamma}} v_{\beta \dot{\gamma}} & =0, \\
\varkappa_{\alpha}^{\gamma} v_{\gamma \dot{\beta}} & =\bar{\varkappa}_{\dot{\beta}}^{\dot{\gamma}} v_{\alpha \dot{\gamma}}, \\
\bar{\varkappa}_{\dot{\alpha}} \dot{\gamma} \bar{\varkappa}_{\dot{\gamma} \dot{\beta}}+v^{\gamma}{ }_{\dot{\alpha}} v_{\gamma \dot{\beta}} & =0 .
\end{aligned}
$$

If (3.4) is satisfied then one can show that an $s p(2)$ flat connection shows up

$$
\mathbf{w}_{\alpha \beta}=\omega_{\alpha \beta}-\frac{1}{z} \mathbf{E}_{\alpha \beta}, \quad \overline{\mathbf{w}}_{\dot{\alpha} \dot{\beta}}=\bar{\omega}_{\dot{\alpha} \dot{\beta}}-\frac{1}{z} \mathbf{E}_{\dot{\alpha} \dot{\beta}},
$$

\footnotetext{
${ }^{6}$ Minkowski limit for $K_{A B}$ corresponds to what is known as rank two dual twistor, that generates $D$ type solutions, see e.g., [41].

${ }^{7}$ We change notation of [4] by introducing $z=\frac{1}{r}$.
} 
where by means of (3.5)-(3.7) one introduces metric

$$
k_{\alpha \dot{\alpha}}=-z^{2} \varkappa_{\alpha}^{\beta} v_{\beta \dot{\alpha}}, \quad k_{\alpha}{ }^{\dot{\gamma}} k_{\beta \dot{\gamma}}=\epsilon_{\alpha \beta}, \quad k_{\dot{\alpha}}^{\gamma} k_{\gamma \dot{\beta}}=\epsilon_{\dot{\alpha} \dot{\beta}}
$$

that allows one converting dotted indices into undotted ones and vice versa according to the following convention. For any $A_{\dot{\alpha}}$ we can define

$$
A_{\alpha}:=k_{\alpha}^{\dot{\beta}} A_{\dot{\beta}},
$$

which entails from (3.9)

$$
A_{\dot{\alpha}}=k_{\dot{\alpha}}^{\gamma} A_{\gamma} .
$$

With this conventions one can define one-forms made of vierbein $\mathbf{e}_{\alpha \dot{\beta}}$

$$
\begin{gathered}
\mathbf{E}_{\alpha \beta}=\frac{z}{2}\left(\mathbf{e}_{\alpha \beta}+\mathbf{e}_{\beta \alpha}\right), \\
\mathbf{E}=z \mathbf{e}_{\alpha}{ }^{\alpha} .
\end{gathered}
$$

The result of these definitions is the $s p(2)$ flatness condition for (3.8)

$$
\mathrm{d} \mathbf{w}_{\alpha \beta}-\mathbf{w}_{\alpha}{ }^{\gamma} \mathbf{w}_{\beta \gamma}=0, \quad \mathrm{~d} \overline{\mathbf{w}}_{\dot{\alpha} \dot{\beta}}-\overline{\mathbf{w}}_{\dot{\alpha}}{ }^{\dot{\gamma}} \overline{\mathbf{w}}_{\dot{\beta} \dot{\gamma}}=0 .
$$

It is natural then to define the following differential

$$
\nabla A_{\alpha \dot{\alpha}}=\mathrm{d} A_{\alpha \dot{\alpha}}-\mathbf{w}_{\alpha}{ }^{\beta} A_{\beta \dot{\alpha}}-\overline{\mathbf{w}}_{\dot{\alpha}}^{\dot{\beta}} A_{\alpha \dot{\beta}}, \quad \nabla^{2}=0,
$$

which provides

$$
\nabla\left(z \varkappa_{\alpha \beta}\right)=\nabla\left(z v_{\alpha \dot{\beta}}\right)=\nabla\left(z \bar{\varkappa}_{\dot{\alpha} \dot{\beta}}\right)=\nabla k_{\alpha \dot{\beta}}=0
$$

and

$$
\nabla \mathbf{E}_{\alpha \beta}=\nabla \mathbf{E}=0, \quad \mathrm{~d} z=\mathbf{E} .
$$

Let us note that the only Lorentz scalar one can construct for the planar case out of components (3.1) is $z,(3.4)$. However, one can define a d-exact 1-form

$$
\mathrm{d} t=-\frac{z}{2} \varkappa_{\alpha \beta} \mathbf{E}^{\alpha \beta}=\frac{1}{2} \mathbf{e}^{\alpha \dot{\alpha}} v_{\alpha \dot{\alpha}},
$$

which has the meaning of time differential as we can see using the Poincaré chart. It should be stressed though, that $t$ itself can not be expressed via $K_{A B}$.

Poincaré realization. The form of global symmetry parameter $K_{A B}(3.1)$ is particularly simple in Poincaré coordinates $(t, x, y, z)$

$$
d s^{2}=\frac{1}{z^{2}}\left(-\mathrm{d} t^{2}+\mathrm{d} x^{2}+\mathrm{d} y^{2}+\mathrm{d} z^{2}\right) .
$$

Let us choose vierbein

$$
\mathbf{e}^{0}=\frac{\mathrm{d} t}{z}, \quad \mathbf{e}^{1}=\frac{\mathrm{d} z}{z}, \quad \mathbf{e}^{2}=\frac{\mathrm{d} x}{z}, \quad \mathbf{e}^{3}=\frac{\mathrm{d} y}{z},
$$


which in spinor terms is given by

$$
\mathbf{e}^{\alpha \dot{\beta}}=\frac{1}{z}\left(\begin{array}{cc}
\mathrm{d} t+\mathrm{d} y & \mathrm{~d} z-i \mathrm{~d} x \\
\mathrm{~d} z+i \mathrm{~d} x & \mathrm{~d} t-\mathrm{d} y
\end{array}\right)
$$

Then Killing vector

$$
v^{\mu}=(1,0,0,0)
$$

generates the following Lorentz components of our global symmetry parameter $K_{A B}$, [52]

$$
v_{\alpha \dot{\beta}}=\frac{1}{z}\left(\begin{array}{ll}
1 & 0 \\
0 & 1
\end{array}\right), \quad \varkappa_{\alpha \beta}=\frac{1}{z}\left(\begin{array}{cc}
1 & 0 \\
0 & -1
\end{array}\right), \quad \bar{\varkappa}_{\dot{\alpha} \dot{\beta}}=\frac{1}{z}\left(\begin{array}{cc}
1 & 0 \\
0 & -1
\end{array}\right) .
$$

Written down in the Poincaré coordinates it has the form

$$
K_{A B}=\frac{1}{z}\left(\begin{array}{cccc}
1 & 0 & 1 & 0 \\
0 & -1 & 0 & 1 \\
1 & 0 & 1 & 0 \\
0 & 1 & 0 & -1
\end{array}\right)
$$

manifestly satisfying (3.4). Note, that definition of $z$ (3.4) literally reproduces the radial Poincaré coordinate, while (3.18) gives Poincaré time differential $\mathrm{d} t$. We are not going to use explicit coordinates anywhere in our paper though leaving them here as a useful illustration.

\section{2 $\nabla$-covariant form of HS equations and the current ansatz}

Let us now bring covariant differential (3.15) into the twisted adjoint sector of HS equations (2.21), (2.23). For that matter we should first rewrite it in terms of oscillator action

$$
\nabla=\mathrm{d}+\mathbf{w}^{\alpha \beta} y_{\alpha} \frac{\partial}{\partial y^{\beta}}-\overline{\mathbf{w}}^{\alpha \beta} \bar{y}_{\alpha} \frac{\partial}{\partial \bar{y}^{\beta}}, \quad \nabla^{2}=0,
$$

where $\mathbf{w}_{\alpha \beta}$ and $\overline{\mathbf{w}}_{\alpha \beta}$ are given in (3.8). Using (2.22) we then obtain

$$
D=\nabla+\frac{1}{z} \mathbf{E}^{\alpha \beta}\left(y_{\alpha} \frac{\partial}{\partial y^{\beta}}-\bar{y}_{\alpha} \frac{\partial}{\partial \bar{y}^{\beta}}\right) .
$$

Substituting this into (2.21) gives

$$
\nabla \mathcal{C}+\frac{i}{z} \mathbf{E}^{\alpha \beta}(y+i \bar{\partial})_{\alpha}(\bar{y}-i \partial)_{\beta} \mathcal{C}+\frac{i}{2 z} \mathbf{E}\left(y_{\alpha} \bar{y}^{\alpha}+\partial_{\alpha} \bar{\partial}^{\alpha}\right) \mathcal{C}=0 .
$$

An important observation that makes problem of non-linear corrections especially interesting is the HS Fock projector that appears in the linearized solution uniformly for all spins [4]. This leads us to introduce the exponential that satisfies the following projector condition

$$
4 e^{i y_{\alpha} \bar{y}^{\alpha}} * 4 e^{i y_{\alpha} \bar{y}^{\alpha}}=4 e^{i y_{\alpha} \bar{y}^{\alpha}},
$$

which in addition is covariantly constant

$$
\nabla e^{i y_{\alpha} \bar{y}^{\alpha}}=0
$$


Fock projector naturally appears in bulk-boundary analysis of HS equations [48] and as shown in [49] decouples from second order equation (2.23) reducing an infinite dimensional twisted-adjoint module to a certain current-type equation that admits polynomial solutions. Given these facts it is natural to adapt HS equations by making them covariant under flat connection (3.8) and proceed along the lines of $[48,49]$ at higher orders by introducing the current ansatz. To this end let us define a current module $\mathcal{T}(w, \bar{w} ; k, \bar{k} \mid x)$ via the following condition

$$
\mathcal{C}=z e^{i y_{\alpha} \bar{y}^{\alpha}} \mathcal{T}(w, \bar{w} ; k, \bar{k} \mid x)
$$

where

$$
w_{\alpha}=\sqrt{z} y_{\alpha}, \quad \bar{w}_{\alpha}=\sqrt{z} \bar{y}_{\alpha} .
$$

Feeding (3.30) into (3.27) and using (3.29) results in the following linear equation for current $\mathcal{T}$

$$
\nabla \mathcal{T}+i \mathbf{E}^{\alpha \beta} \partial_{\alpha} \bar{\partial}_{\beta} \mathcal{T}+\frac{i}{2} \mathbf{E} \partial_{\alpha} \bar{\partial}^{\alpha} \mathcal{T}=0
$$

where $\partial_{\alpha}$ and $\bar{\partial}_{\alpha}$ are partial derivatives with respect to $w$ and $\bar{w}$ correspondingly, while covariant differential (3.25) is redefined to act properly on functions of $w$ and $\bar{w}$

$$
\nabla:=\mathrm{d}+\mathbf{w}^{\alpha \beta} w_{\alpha} \frac{\partial}{\partial w^{\beta}}-\overline{\mathbf{w}}^{\alpha \beta} \bar{w}_{\alpha} \frac{\partial}{\partial \bar{w}^{\beta}}, \quad \mathrm{d} w_{\alpha}=\mathrm{d} \bar{w}_{\alpha}=0 .
$$

Remarkable property of ansatz (3.30) is that it goes through nonlinear HS equation (2.23) factoring out Fock exponential $e^{i y_{\alpha} \bar{y}^{\alpha}}$ from the nonlinear right hand side. This phenomenon takes place due to a somewhat specific form of the interaction term in (2.23). In particular, one may note that Fock projector (3.28) being rescaled by the homotopy integration variable $\tau$ as prescribed by (2.23) commutes with operator $(1-\tau) \partial_{1}-\tau \partial_{2}$ that enters the quadratic vertex. Substituting (3.30) into (2.23) and after some simple algebra that includes change of integration variables one arrives at

$$
\begin{aligned}
\nabla \mathcal{T}+i \mathbf{E}^{\alpha \beta} \partial_{\alpha} \bar{\partial}_{\beta} \mathcal{T}+\frac{i}{2} \mathbf{E} \partial_{\alpha} \bar{\partial}^{\alpha} \mathcal{T} & =\mathbb{J}^{c}+\mathbb{J}^{\omega}, \\
\mathbb{J}^{c}[\mathcal{T}, \mathcal{T}] & =\mathbf{E}^{\alpha \beta} \mathcal{J}_{\alpha \beta}[\mathcal{T}, \mathcal{T}]+\mathbf{E} \mathcal{J}[\mathcal{T}, \mathcal{T}]
\end{aligned}
$$

where $\mathbb{J}^{c}$ stems from the current interaction in the second line of (2.23), while $\mathbb{J}^{\omega}$ is the gauge part of commutator $[\mathbf{w}, C]_{*}$. Explicitly, current contribution $\mathbb{J}^{c}$ has the form

$$
\begin{aligned}
\mathcal{J}_{\alpha \beta}= & -\frac{\eta}{4 z^{2}} w_{\alpha} \int \frac{\mathrm{d} \bar{u} \mathrm{~d} \bar{v}}{(2 \pi)^{2}} \int_{0}^{1} \mathrm{~d} \tau e^{-\frac{i}{z} \bar{u}_{\alpha} \bar{v}^{\alpha}}\left((1-\tau) \partial_{\bar{u}}-\tau \partial_{\bar{v}}\right)_{\beta} \\
& \times \mathcal{T}(\tau w, \bar{u}+\bar{w}+(1-\tau) w) \mathcal{T}(-(1-\tau) w, \bar{v}+\bar{w}-\tau w) k+(\alpha \leftrightarrow \beta) \\
& +\frac{\bar{\eta}}{4 z^{2}} \bar{w}_{\alpha} \int \frac{\mathrm{d} u \mathrm{~d} v}{(2 \pi)^{2}} \int_{0}^{1} \mathrm{~d} \tau e^{\frac{i}{z} u_{\alpha} v^{\alpha}}\left((1-\tau) \partial_{u}-\tau \partial_{v}\right)_{\beta} \\
& \times \mathcal{T}(u+w+(1-\tau) \bar{w}, \tau \bar{w}) \mathcal{T}(v+w-\tau \bar{w},-(1-\tau) \bar{w}) \bar{k}-(\alpha \leftrightarrow \beta),
\end{aligned}
$$




$$
\begin{aligned}
\mathcal{J}= & \frac{\eta}{4 z^{2}} w^{\alpha} \int \frac{\mathrm{d} \bar{u} \mathrm{~d} \bar{v}}{(2 \pi)^{2}} \int_{0}^{1} \mathrm{~d} \tau e^{-\frac{i}{z} \bar{u}_{\alpha} \bar{v}^{\alpha}}\left((1-\tau) \partial_{\bar{u}}-\tau \partial_{\bar{v}}\right)_{\alpha} \\
& \times \mathcal{T}(\tau w, \bar{u}+\bar{w}+(1-\tau) w) \mathcal{T}(-(1-\tau) w, \bar{v}+\bar{w}-\tau w) k \\
& +\frac{\bar{\eta}}{4 z^{2}} \bar{w}^{\alpha} \int \frac{\mathrm{d} u \mathrm{~d} v}{(2 \pi)^{2}} \int_{0}^{1} \mathrm{~d} \tau e^{\frac{i}{z} u_{\alpha} v^{\alpha}}\left((1-\tau) \partial_{u}-\tau \partial_{v}\right)_{\alpha} \\
& \times \mathcal{T}(u+w+(1-\tau) \bar{w}, \tau \bar{w}) \mathcal{T}(v+w-\tau \bar{w},-(1-\tau) \bar{w}) \bar{k}
\end{aligned}
$$

while gauge part $\mathbb{J}^{\omega}$ is

$$
\begin{aligned}
\mathbb{J}^{\omega}= & \frac{1}{(2 \pi)^{4} z^{4}} \int \mathrm{d} u \mathrm{~d} v \mathrm{~d} \bar{u} \mathrm{~d} \bar{v} e^{\frac{i}{z}\left(u_{\alpha} v^{\alpha}-\bar{u}_{\alpha} \bar{v}^{\alpha}\right)} \times \\
& \times(\mathcal{T}(w+u, \bar{u}+\bar{w}) \mathbf{w}(v-\bar{u}+w-\bar{w}, \bar{v}+\bar{w}-w) \\
& -\mathbf{w}(u+w+\bar{w}, \bar{u}+v+w+\bar{w}) \mathcal{T}(v+w, \bar{w}+\bar{v})) .
\end{aligned}
$$

We warn the reader on manipulations with Klein operators $k$ and $\bar{k}$ that fields $\mathcal{T}$ and $\mathbf{w}$ implicitly contain in the above formulae. When dragging, say, $k$ to the right through a master field be it $\mathcal{T}$ or $\mathbf{w}$ one should change the sign of its whole first spinorial argument. Similarly for $\bar{k}$ and the second argument. In other words, one should use the following rule, e.g., $k \mathcal{T}(a, b)=\mathcal{T}(-a, b) k$.

\section{Solutions}

Recall that our goal is to solve (2.23) which reads schematically

$$
D \mathcal{C}^{(2)}-i \mathbf{e}^{\alpha \dot{\alpha}}\left(y_{\alpha} \bar{y}_{\dot{\alpha}}-\frac{\partial}{\partial y^{\alpha}} \frac{\partial}{\partial \bar{y}^{\dot{\alpha}}}\right) \mathcal{C}^{(2)}=\mathbf{J}\left(\mathcal{C}^{(\mathbf{1})}, \mathcal{C}^{(\mathbf{1})}\right),
$$

where $\mathcal{C}^{(1)}$ comes from the first order solution sourcing $\mathcal{C}^{(2)}$ at the second. Note that $\mathbf{J}(\mathcal{C}, \mathcal{C})$ contains contribution from two pieces, the current-type on the second line of (2.23) and the gauge one $[\mathbf{w}, \mathcal{C}]$, which is also of $\mathcal{C C}$-type, since $\mathbf{w}=\mathbf{w}[\mathcal{C}]$. The source is made of linearized solution [4]. It is static and has two-dimensional planar symmetry. We are interested in quadratic corrections $\mathcal{C}^{(2)}$ that maintain this symmetry. To find these we therefore need explicit form of $\mathbf{J}\left(\mathcal{C}^{(\mathbf{1})}, \mathcal{C}^{(\mathbf{1})}\right)$. It is much simpler however analyzing (3.34) and for that the free solution should be taken from (3.32). Since the linearized solution of [4] was obtained for HS $A$-model from now on we set

$$
\eta=\bar{\eta}=1
$$

\subsection{Linear solutions}

At free level the final result of [4] that solves (2.21) and (2.19) is ${ }^{8}$

$$
\begin{aligned}
C^{(1)} & =z(f(y)+f(\bar{y})) e^{i y_{\alpha} \bar{y}^{\alpha},} \\
\mathbf{w}^{(1)} & =-\frac{i}{2} \mathbf{E}^{\alpha \beta} \int_{0}^{1} \mathrm{~d} \tau \partial_{\alpha} \partial_{\beta} f(\tau y+(1-\tau) \bar{y}),
\end{aligned}
$$

\footnotetext{
${ }^{8}$ Note that the exponential projector factor in $C$ is unique for any spin $s$. This behavior is typical of HS bulk-to-boundary propagators $[54,55]$ that contain similar projector for every spin in the twisted-adjoint.
} 
where

$$
f(y):=f\left(\frac{z^{2}}{2} \varkappa_{\alpha \beta} y^{\alpha} y^{\beta}\right), \quad f(\bar{y}):=f\left(\frac{z^{2}}{2} \varkappa_{\alpha \beta} \bar{y}^{\alpha} \bar{y}^{\beta}\right)
$$

is an arbitrary function of its bilinear in $y$ 's argument and $\partial_{\alpha}$ in (4.4) differentiates a spinorial argument. Eqs. (4.3) and (4.4) describe bosonic fields only. For any $s>0$ the solution is static and possesses planar symmetry. ${ }^{9}$ For example, $s=2$ component in (4.3) corresponds to the Weyl tensor of a black brane. The whole freedom of the solution is stored in the Taylor coefficients of function $f(x)$, which are associated with spin $s$ parameters. A particular example of $f_{s}(x)$ that describes a single spin $s$ field is

$$
f_{s}(y)=\frac{m_{s}}{s !}\left(\frac{z^{2}}{2} \varkappa_{\alpha \beta} y^{\alpha} y^{\beta}\right)^{s}
$$

where $m_{s}$ is an arbitrary real number. In fact, $f$ plays a role of a generating function for the primary HS Weyl tensors of the form

$$
C_{\alpha_{1} \ldots \alpha_{2 s}}^{(1)}=m_{s} z^{2 s+1} \varkappa_{\left(\alpha_{1} \alpha_{2}\right.} \cdots \varkappa_{\left.\alpha_{2 s-1} \alpha_{2 s}\right)} .
$$

Therefore, $f$ is no more than polynomial for a finite set of spins, despite the fact that the twisted-adjoint module $\mathcal{C}$ is infinite dimensional. Note that (4.7) are of Petrov $D$ type and as pointed out in [4] have multi-copy origin.

The scalar case $s=0$ corresponds to

$$
C_{\Delta=1}^{(1)}=m_{0} z e^{i y_{\alpha} \bar{y}^{\alpha}}
$$

which is the solution with $\Delta=1$ boundary $z=0$ asymptotics. Note, that (4.3) contains no scalar with alternative boundary condition $\Delta=2$. It was also found in [4] in the form

$$
C_{\Delta=2}^{(1)}=m_{0}^{\prime} z^{2}\left(1+i y_{\alpha} \bar{y}^{\alpha}\right) e^{i y_{\alpha} \bar{y}^{\alpha}} .
$$

We exclude $\Delta=2$ contribution from consideration by setting $m_{0}^{\prime}=0$ for simplicity. It is important to stress that (4.3), (4.4) is a solution of the type- $A$ HS model (4.2) in (2.19) and at higher orders (2.23) and is not applicable for general $\eta$.

In terms of current module (3.30), solution (4.3) and (4.4) corresponds to the following (anti)holomorphic currents

$$
\begin{aligned}
& \mathcal{T}_{(1)}^{b}=(f(w)+f(\bar{w}))(k+\bar{k}), \\
& \mathcal{T}_{(1)}^{h}=f(w) k+f(\bar{w}) \bar{k},
\end{aligned}
$$

where

$$
f(w):=f\left(\frac{z}{2} \varkappa_{\alpha \beta} w^{\alpha} w^{\beta}\right), \quad f(\bar{w}):=f\left(\frac{z}{2} \varkappa_{\alpha \beta} \bar{w}^{\alpha} \bar{w}^{\beta}\right)
$$

and $\mathcal{T}_{(1)}^{b}$ gives the standard bosonic embedding (2.24), while $\mathcal{T}_{(1)}^{h}$ the chiral one (2.30). Note that (4.10) and (4.11) both being the sum of holomorphic and antiholomorphic functions

\footnotetext{
${ }^{9}$ The case of $s=0$ is special since it has larger global symmetry which is the boundary Poincaré algebra.
} 
trivially solve (3.32). Indeed, since $\nabla f(w)=\nabla f(\bar{w})=0$, (3.32) is satisfied because it contains mixed derivatives. Gauge 1-form $\mathbf{w}$ is expressed via (4.4) for both embeddings as follows

$$
\mathbf{w}_{(1)}^{b, h}=-\frac{i}{2} \mathbf{E}^{\alpha \beta} \int_{0}^{1} \mathrm{~d} \tau \partial_{\alpha} \partial_{\beta} f(\tau w+(1-\tau) \bar{w})(1+k \bar{k})
$$

with $f(w)$ given by (4.12). Note, that in terms of currents solution (4.3) looks very simple being just power $s$ of covariantly constant $\frac{z}{2} \varkappa_{\alpha \beta} w^{\alpha} w^{\beta}$ for spin $s$.

\subsection{Quadratic analysis}

Second order corrections of the twisted-adjoint sector are governed by (3.34), where its right hand side should be computed on free solutions (4.10) and (4.13) in the bosonic case and (4.11), (4.13) in the case of chiral embedding. This implies that quadratic source $\mathbb{J}^{c}\left(\mathcal{T}^{(1)}, \mathcal{T}^{(1)}\right)+\mathbb{J}^{\omega}\left(\mathcal{T}^{(1)}, \mathbf{w}^{(1)}\right)$ is totally defined in terms of function $f(x)$. Let us calculate it in the bosonic case (4.10). For that we take

$$
\mathcal{T}_{(2)}^{b}=T(w, \bar{w} ; z)(k+\bar{k}) .
$$

Substituting (4.14) into the left hand side of (3.34) while (4.10) and (4.13) into the right one and after straightforward calculation we arrive at

$$
\nabla T+i \mathbf{E}^{\alpha \beta} \partial_{\alpha} \bar{\partial}_{\beta} T+\frac{i}{2} \mathbf{E} \partial_{\alpha} \bar{\partial}^{\alpha} T=\mathbf{E}^{\alpha \beta} J_{\alpha \beta}+\mathbf{E} J,
$$

where

$$
J_{\alpha \beta}=J_{\alpha \beta}^{c}+J_{\alpha \beta}^{\omega} .
$$

$J_{\alpha \beta}^{c}$ and $J$ come from current terms (3.36) and (3.37) correspondingly

$$
\begin{aligned}
J_{\alpha \beta}^{c}= & \frac{1}{2 z^{2}} \int \frac{\mathrm{d} u \mathrm{~d} v}{(2 \pi)^{2}} \int_{0}^{1} \mathrm{~d} \tau e^{\frac{i}{z} u_{\alpha} v^{\alpha}}\left[-w_{\alpha} \frac{\partial}{\partial w^{\beta}}(f(v+\bar{w}+(1-\tau) w) f(u+\bar{w}-\tau w))\right. \\
& \left.+\bar{w}_{\alpha} \frac{\partial}{\partial \bar{w}^{\beta}}(f(u+y+(1-\tau) \bar{w}) f(v+w-\tau \bar{w}))\right] \\
& +\frac{1}{2} \int_{0}^{1} \mathrm{~d} \tau\left[-w_{\alpha} f(\tau y) \frac{\partial}{\partial w^{\beta}}(f(\bar{w}+\tau w)+f(\bar{w}-\tau w))\right. \\
& \left.+\bar{w}_{\alpha} f(\tau \bar{w}) \frac{\partial}{\partial \bar{w}^{\beta}}(f(w+\tau \bar{w})+f(w-\tau \bar{w}))\right]+(\alpha \leftrightarrow \beta)
\end{aligned}
$$

and

$$
\begin{aligned}
J= & \frac{1}{2 z^{2}} \int \frac{\mathrm{d} u \mathrm{~d} v}{(2 \pi)^{2}} \int_{0}^{1} \mathrm{~d} \tau e^{\frac{i}{z} u_{\alpha} v^{\alpha}}\left[w^{\alpha} \frac{\partial}{\partial w^{\alpha}}(f(v+\bar{w}+(1-\tau) w) f(u+\bar{w}-\tau w))\right. \\
& +\bar{w}^{\alpha} \frac{\partial}{\partial \bar{w}^{\alpha}}(f(u+w+(1-\tau) \bar{w}) f(v+w-\tau \bar{w})) \\
& +z^{2} f(\tau w) w^{\alpha} \frac{\partial}{\partial w^{\alpha}}(f(\bar{w}+\tau w)+f(\bar{w}-\tau w)) \\
& \left.+z^{2} f(\tau \bar{w}) \bar{w}^{\alpha} \frac{\partial}{\partial \bar{w}^{\alpha}}(f(w+\tau \bar{w})+f(w-\tau \bar{w}))\right]
\end{aligned}
$$


while $J^{\omega}$ comes from (3.39)

$$
\begin{aligned}
J_{\alpha \beta}^{\omega}= & \frac{i}{z} \int \frac{\mathrm{d} u \mathrm{~d} v}{(2 \pi)^{2}} \int_{0}^{1} \mathrm{~d} \tau e^{\frac{i}{z} u_{\alpha} v^{\alpha}} \frac{\partial}{\partial \bar{w}^{\alpha}} \frac{\partial}{\partial \bar{w}^{\beta}}(f(v+w)(f(w+\bar{w}+\tau u)-f(\bar{w}-w+\tau u))) \\
& +e^{-\frac{i}{z} \bar{u}_{\alpha} \bar{v}^{\alpha}} \frac{\partial}{\partial w^{\alpha}} \frac{\partial}{\partial w^{\beta}}(f(\bar{v}+\bar{w})(f(w+\bar{w}+\tau \bar{u})-f(w-\bar{w}+\tau \bar{u}))) .
\end{aligned}
$$

In obtaining the above expressions we use that $f(y)$ is even and also change integration variable $\tau \rightarrow 1-\tau$ in certain terms. Compatibility condition $\nabla^{2}=0$ for (4.15) gives the following constraint on the interaction terms

$$
\frac{i}{2}\left(\mathbf{E}^{\alpha \beta} \partial_{\alpha} \bar{\partial}_{\beta}+\mathbf{E} \partial_{\alpha} \bar{\partial}^{\alpha}\right)\left(\mathbf{E}^{\gamma \delta} J_{\gamma \delta}+\mathbf{E} J\right)=-\mathbf{E}^{\alpha \beta} \nabla J_{\alpha \beta},
$$

where in obtaining this result we applied $\nabla$ to (4.15) and noted that

$$
\mathbf{E} \nabla J=0 .
$$

The latter property is due to the fact that since $\nabla f=0, \nabla$ acts on (4.18) nontrivially in its manifest $z$-dependence only. The result of that action is therefore proportional to $\nabla z=\mathbf{E}$ and since $\mathbf{E}^{2}=0$, (4.21) holds. Using the Schouten identity

$$
\mathbf{E}^{\alpha \beta} \mathbf{E}^{\gamma \delta}=\frac{1}{2} \mathbf{E}_{\mu}^{\alpha} \mathbf{E}^{\mu \delta} \epsilon^{\beta \gamma}+\frac{1}{2} \mathbf{E}_{\mu}^{\beta} \mathbf{E}^{\mu \gamma} \epsilon^{\alpha \delta}
$$

one rewrites (4.20) further as

$$
\begin{aligned}
\partial_{\gamma} \bar{\partial}_{\alpha} J_{\beta}^{\gamma}+\bar{\partial}_{\gamma} \partial_{\alpha} J_{\beta}^{\gamma}=0, \\
\frac{i}{2} \mathbf{E}\left(\partial_{\alpha} \bar{\partial}_{\alpha} J-\partial_{\beta} \bar{\partial}^{\beta} J_{\alpha \alpha}\right)=\nabla J_{\alpha \alpha} .
\end{aligned}
$$

Eq. (4.23) and (4.24) are satisfied as a result of the consistency of HS equation (2.23) and needs no verification.

\subsubsection{Static condition}

Let us now note, that since we are interested in the deformation of the linearized solution (4.10) by HS interactions, the former should depend on the same set of fields as the latter. This implies that the corresponding $T$ in (4.14) being a solution of (4.15) should depend on the Lorentz components of the global symmetry parameter $K_{A B}$, (3.1) only, much as the free solution does. Those are the $\nabla$-covariantly constant upon proper rescaling fields (3.16) and Lorentz scalar $z$. Thus, the only allowed space-time dependence for $T$ which is not constant with respect to $\nabla$ is via variable $z$. This guarantees that the deformed solution maintains planar and static symmetry. In particular, there must be no dependence on time $t$ (3.18) in $T$ either which is the static requirement. Practically this means that $\nabla T \sim \mathbf{E}$ contains no one-forms $\mathbf{E}_{\alpha \beta}$. This in turn implies that (4.15) can be equivalently rewritten as

$$
\begin{aligned}
\frac{1}{2}\left(\partial_{\alpha} \bar{\partial}_{\beta}+\partial_{\beta} \bar{\partial}_{\alpha}\right) T & =-i J_{\alpha \beta}, \\
\left(\nabla+\frac{i}{2} \mathbf{E} \partial_{\alpha} \bar{\partial}^{\alpha}\right) T & =\mathbf{E} J .
\end{aligned}
$$


Eq. (4.25) governs the dependence of $T$ on $\nabla$-constant fields, while (4.26) its radial dependence $^{10}$ on $z$. Note that (4.25) places stringent constraints on possible HS interactions that respect nonlinear deformation (4.14) and will be called the static condition. Its relation to static symmetry is as follows. Suppose $T$ depends also on time, i.e., $T=T(w, \bar{w} ; z, t)$. In that case property $\nabla T \sim \mathbf{E}$ is no longer true, since $\nabla T=\frac{\partial}{\partial z} T \mathbf{E}+\frac{\partial}{\partial t} T \mathrm{~d} t$, where $\mathrm{d} t$ is given by (3.18), which contains $\mathbf{E}^{\alpha \beta}$-part. So, one can not separate (4.15) into pieces (4.25) and (4.26).

HS interactions that are consistent with (4.25) should satisfy the following compatibility conditions

$$
\begin{gathered}
\partial_{\gamma} \bar{\partial}_{\alpha} J_{\beta}^{\gamma}+\bar{\partial}_{\gamma} \partial_{\alpha} J_{\beta}^{\gamma}=0, \\
\partial_{\alpha} \partial_{\beta} J^{\alpha \beta}=\bar{\partial}_{\alpha} \bar{\partial}_{\beta} J^{\alpha \beta}=0 .
\end{gathered}
$$

While (4.27) is already on the list (4.23) being a part of general HS consistency, it is not granted that (4.28) is satisfied and therefore should be verified separately. Verification of (4.28) being quite tedious is the most challenging part of this paper. The important result that ensures the existence of planar solution is that (4.28) does hold for bosonic embedding (4.14). For chiral embedding (4.11) static constraint (4.28) turns out to be not satisfied. We interpret this result as there is no quadratic deformation of the linearized solution (4.11) which is both static and planar in the chiral case. Though rather straightforward, the check of (4.28) involves few partial integrations and we leave it for appendix.

\subsection{Solving the bosonic embedding}

Once (4.28) is shown to be satisfied for bosonic embedding (4.14), we can try to solve (4.25) and (4.26) and find this way the nonlinear deformation of planar solution (4.10) in the sector of HS curvatures. To do so we note that (4.25) can be rewritten as

$$
\partial_{\alpha} \bar{\partial}_{\beta} T=-i J_{\alpha \beta}+i \epsilon_{\alpha \beta} h:=X_{\alpha \beta},
$$

where $h=h(w, \bar{w} ; z)$ is some unknown function. Indeed, (4.25) says that the symmetrized derivatives acting on $T$ gives $J_{\alpha \beta}$. This statement is equivalent to the following. Relaxing the symmetrization of the derivatives one still reproduces $J_{\alpha \beta}$ up to something which is skew symmetric $h_{\alpha \beta}=-h_{\beta \alpha}$. But since spinorial indices range only two values, $h_{\alpha \beta} \sim \epsilon_{\alpha \beta}$ and therefore we have (4.29). Function $h$ can not be arbitrary however as it is constrained by the following integrability conditions

$$
\partial_{\alpha} h=-\partial_{\beta} J_{\alpha}{ }^{\beta}, \quad \bar{\partial}_{\alpha} h=\bar{\partial}_{\beta} J_{\alpha}{ }^{\beta} .
$$

Consistency of (4.30) in turn is fulfilled by (4.28). The general solution of (4.29) can be written down by applying two consecutive contracting homotopies

$$
T=\int_{[0,1]^{2}} \mathrm{~d} \tau \mathrm{d} \bar{\tau} w^{\alpha} \bar{w}^{\beta} X_{\alpha \beta}(\tau w, \bar{\tau} \bar{w} ; z)+\phi(w ; z)+\bar{\phi}(\bar{w} ; z),
$$

\footnotetext{
${ }^{10}$ To be a bit more specific, using the Poincaré coordinates (3.19) one shows that (4.25) defines behavior on boundary coordinates $x^{i}$ in a way consistent with planar symmetry, while (4.26) sets evolution inside the bulk along $z$.
} 
where $\phi(w ; z)$ and $\bar{\phi}(\bar{w} ; z)$ are arbitrary at this stage (anti)holomorphic functions. The $\phi$-part of $T$ in (4.31) encodes HS Weyl tensors that are extracted from $T(w, 0 ; z)$ and $T(0, \bar{w} ; z)$, while the $X$-part corresponds to their on-shell derivatives. Hence, physical information about the nonlinear HS deformation is stored in yet unknown $\phi$-functions. Note that the scalar contribution to $T$ is placed within sum $\phi(0, z)+\bar{\phi}(0, z)$ rather than in the individual pieces $\phi(0, z)$ and $\bar{\phi}(0, z)$. This allows one to set

$$
\phi(0, z)=\bar{\phi}(0, z)
$$

for convenience. The solution of (4.29) can be explicitly found once $h(w, \bar{w} ; z)$ is known. To restrict the latter one can again use contracting homotopy in both equations (4.30). This will fix $h(w, \bar{w} ; z)$ up to a $w$ and $\bar{w}$-independent function of $z$. Indeed, from first equation in (4.30) one finds

$$
h=-\int_{0}^{1} \frac{\mathrm{d} \tau}{\tau} w^{\alpha} \frac{\partial}{\partial w^{\beta}} J_{\alpha}^{\beta}(\tau w, \bar{w})+\bar{g}(\bar{w} ; z),
$$

while from the second,

$$
h=\int_{0}^{1} \frac{\mathrm{d} \tau}{\tau} \bar{w}^{\alpha} \frac{\partial}{\partial \bar{w}^{\beta}} J_{\alpha}^{\beta}(w, \tau \bar{w})+g(w ; z),
$$

where $g(w ; z)$ and $\bar{g}(\bar{w} ; z)$ are so far unspecified functions. Since (4.33) and (4.34) should give one and the same result for $h(w, \bar{w} ; z)$ by equating the two expressions and setting either $w$ or $\bar{w}$ to zero we fix $g$ and $\bar{g}$ up to an arbitrary constant $\mathbf{c}(z)$ implying the following final result

$$
h=\int_{0}^{1} \frac{\mathrm{d} \tau}{\tau}\left(\bar{w}^{\alpha} \frac{\partial}{\partial \bar{w}^{\beta}} J_{\alpha}^{\beta}(w, \tau \bar{w} ; z)-w^{\alpha} \frac{\partial}{\partial w^{\beta}} J_{\alpha}^{\beta}(\tau w, 0 ; z)\right)+\mathbf{c}(z)
$$

or equivalently

$$
h=\int_{0}^{1} \frac{\mathrm{d} \tau}{\tau}\left(-w^{\alpha} \frac{\partial}{\partial w^{\beta}} J_{\alpha}^{\beta}(\tau w, \bar{w} ; z)+\bar{w}^{\alpha} \frac{\partial}{\partial \bar{w}^{\beta}} J_{\alpha}^{\beta}(0, \tau \bar{w} ; z)\right)+\mathbf{c}(z) .
$$

That (4.35) and (4.36) are identical is guaranteed by consistency condition (4.28). The result of the analysis of (4.25) allows one fixing $T$ up to an arbitrary holomorphic function $\phi(w ; z)$ (and its complex conjugate) and $w, \bar{w}$-independent function $\mathbf{c}(z)$. Explicit expression for $h(w, \bar{w})$ is provided in the appendix.

\subsubsection{Determining $\mathrm{c}(z)$}

It is not difficult to obtain equation on $\mathbf{c}(z)=h(0,0 ; z)$. To do so we first note that from (4.29) it follows

$$
\partial_{\alpha} \bar{\partial}^{\alpha} T=2 i h .
$$

Acting then on (4.29) with $\nabla$ and using (4.26) one gets

$$
\mathbf{E} \partial_{\alpha} \bar{\partial}_{\beta}\left(J-\frac{i}{2} \partial_{\gamma} \bar{\partial}^{\gamma} T\right)=i \nabla\left(-J_{\alpha \beta}+\epsilon_{\alpha \beta} h\right),
$$


which upon substitution (4.37) gives

$$
\mathbf{E} \partial_{\alpha} \bar{\partial}_{\beta}(J+h)=i \nabla\left(-J_{\alpha \beta}+\epsilon_{\alpha \beta} h\right) .
$$

Contracting indices in (4.39) and using that $J_{\alpha \beta}=J_{\beta \alpha}$ we then have

$$
\mathbf{E} \partial_{\alpha} \bar{\partial}^{\alpha}(J+h)=2 i \nabla h .
$$

From (4.30) one extracts

$$
\partial_{\alpha} \bar{\partial}^{\alpha} h=-\partial_{\alpha} \bar{\partial}_{\beta} J^{\alpha \beta}
$$

which yields

$$
\nabla h=\frac{1}{2 i} \mathbf{E}\left(\partial_{\alpha} \bar{\partial}^{\alpha} J-\partial_{\alpha} \bar{\partial}_{\beta} J^{\alpha \beta}\right) .
$$

Finally, setting $w_{\alpha}=\bar{w}_{\alpha}=0$ and using (4.35) we arrive at the desired equation for $\mathbf{c}(z)$

$$
\mathbf{c}^{\prime}(z)=\frac{1}{2 i}\left(\partial_{\alpha} \bar{\partial}^{\alpha} J(0,0 ; z)-\partial_{\alpha} \bar{\partial}_{\beta} J^{\alpha \beta}(0,0 ; z)\right) .
$$

The right hand side is readily accessible from (4.17)-(4.19) and can be easily calculated explicitly. Quite interestingly, the result appears to have a neat form of the total derivative with respect to $z$ once one takes into account the specific dependence $f(w)$ on $w$ via a combination (4.12)

$$
x=\frac{z}{2} \varkappa_{\alpha \beta} w^{\alpha} w^{\beta} .
$$

One then finds

$$
\mathbf{c}^{\prime}(z)=2 \frac{\partial}{\partial z} \int \frac{\mathrm{d} u \mathrm{~d} v}{(2 \pi)^{2}} e^{\frac{i}{z} u_{\alpha} v^{\alpha}} f^{\prime}(v) f^{\prime}(u),
$$

where $f^{\prime}(w):=\frac{\partial}{\partial x} f(w)$. Solution for $\mathbf{c}(z)$ has a freedom from homogeneous equation that gives some constant $\mathbf{c}_{0}$. It can be set to zero, because $\mathbf{c}_{0} \neq 0$ corresponds to a freedom in solution of homogeneous field equation (3.32), which has been fixed at free level already. Indeed, as is seen from (4.31), nonzero $\mathbf{c}_{0}$ induces free scalar with $\Delta=2$ boundary behavior (4.9)

$$
T_{\Delta=2}=\mathbf{c}_{0}\left(z+i w_{\alpha} \bar{w}^{\alpha}\right) .
$$

This has been set to zero at free level. As a result,

$$
\mathbf{c}(z)=2 \int \frac{\mathrm{d} u \mathrm{~d} v}{(2 \pi)^{2}} e^{\frac{i}{z} u_{\alpha} v^{\alpha}} f^{\prime}(v) f^{\prime}(u) .
$$

\subsubsection{HS Weyl tensors}

Having found $\mathbf{c}(z)$ we have completely determined function $h$ that enters (4.29) via (4.35). Using this information we can now find the leftover $\phi(w ; z)$ and $\bar{\phi}(\bar{w} ; z)$ from (4.31) thus fully fixing the current module at second order and correspondingly the twisted-adjoint one through (3.30). Recall, that all HS Weyl tensors are stored in $\phi$ 's. To arrive at the equation on $\phi$ we substitute (4.37) into (4.26) to obtain

$$
\nabla T=\mathbf{E}(J+h)
$$


Feeding (4.31) and (4.35), (4.47) into (4.48) one gets pretty complicated equation, which contains all the required information about $\phi$ 's. Equation that determines evolution of $\phi(w ; z)$ along $z$ can be easily extracted from it by setting $\bar{w}_{\alpha}=0$

$$
\partial_{z}(\phi(w ; z)+\bar{\phi}(0 ; z))=J(w, 0 ; z)+h(w, 0 ; z) .
$$

Using now (4.32) and also that $J(0,0 ; z)=0$ one finds

$$
2 \phi^{\prime}(0 ; z)=h(0,0 ; z)=\mathbf{c}(z)
$$

hence,

$$
\partial_{z} \phi(w ; z)=J(w, 0 ; z)+h(w, 0 ; z)-\frac{1}{2} \mathbf{c}(z) .
$$

Similar equation arises for $\bar{\phi}(\bar{w} ; z)$. Upon straightforward if perhaps somewhat annoying calculation one is able to arrive at quite a simple explicit form for the right hand side of (4.51)

$$
\begin{aligned}
& J(w, 0)+h(w, 0)-\frac{1}{2} \mathbf{c}(z) \\
& =\frac{1}{z^{2}} \int \frac{\mathrm{d} u \mathrm{~d} v}{(2 \pi)^{2}} \int_{0}^{1} \mathrm{~d} \tau e^{\frac{i}{z} u_{\alpha} v^{\alpha}}(f(w+u) f(w+v)+f(v) f(u-w)-2 f(u) f(v) \\
& \left.\quad-f(u-\tau w) \partial_{\tau} f(v+(1-\tau) w)+z^{2} f^{\prime}(u) f^{\prime}(v)\right) .
\end{aligned}
$$

Closed form solution of (4.51) can be written down as a homotopy integral

$$
\begin{aligned}
\phi(w, z)= & \int \frac{\mathrm{d} u \mathrm{~d} v}{(2 \pi)^{2}} \int_{[0,1]^{2}} \mathrm{~d} \tau \mathrm{d} \sigma e^{\frac{i}{z \sigma} u_{\alpha} v^{\alpha}} \frac{1}{\sigma^{2} z}(f(w+u) f(w+v)+f(v) f(u-w) \\
& \left.-2 f(u) f(v)-f(u-\tau w) \partial_{\tau} f(v+(1-\tau) w)+\sigma^{2} z^{2} f^{\prime}(u) f^{\prime}(v)\right) .
\end{aligned}
$$

Poles $\frac{1}{\sigma}$ in the exponential and in the prefactor may look worrisome, but in fact the integral is perfectly well defined as can be seen upon changing variables $u \rightarrow \sqrt{\sigma z} u, v \rightarrow \sqrt{\sigma z} v$

$$
\begin{aligned}
\phi(w, z)= & z \int \frac{\mathrm{d} u \mathrm{~d} v}{(2 \pi)^{2}} \int_{[0,1]^{2}} \mathrm{~d} \tau \mathrm{d} \sigma e^{i u_{\alpha} v^{\alpha}} \\
& \times(f(w+u \sqrt{\sigma z}) f(w+v \sqrt{\sigma z})+f(v \sqrt{\sigma z}) f(u \sqrt{\sigma z}-w)-2 f(u \sqrt{\sigma z}) f(v \sqrt{\sigma z}) \\
& \left.-f(\sqrt{\sigma z} u-\tau w) \partial_{\tau} f(\sqrt{\sigma z} v+(1-\tau) w)+\sigma^{2} z^{2} f^{\prime}(\sqrt{\sigma z} u) f^{\prime}(\sqrt{\sigma z} v)\right) .
\end{aligned}
$$

There is a natural freedom in $z$-independent solutions $\phi_{0}(w)$ of homogeneous part of equation (4.51). We set $\phi_{0}(w)=0$ for it corresponds to the solutions of the free HS equations which have been already fixed by (4.10).

The full result for $T,(4.31)$ has a complicated form and is not particularly illuminating to be written down here. Its most important part, however, is stored in (4.53). Indeed, $\phi(w ; z)$ is nothing but the generating function for quadratic corrections to holomorphic components of the HS Weyl tensors as follows from (3.30) for $s>0(y \neq 0)$

$$
C^{(2)}(y, 0 ; x)=z \phi(\sqrt{z} y ; z)
$$

and

$$
C^{(2)}(0,0 ; x)=z(\phi(0 ; z)+\bar{\phi}(0 ; z))=2 z \phi(0 ; z)
$$

for $s=0$. 


\subsection{Properties of the quadratic corrections}

Here we collect some important properties of our solution.

- Algebraically special. Recall that planar solution (4.5) is algebraically special for every spin $s>0,(4.6)$ at the linearized level. This is seen from (4.7), which says that the HS Weyl tensors are of the generalized Petrov $D$-type. The same is true for quadratic deformation. Indeed, by analyzing (4.53) for $f=\sum_{s} f_{s}$, (4.6) one can schematically extract

$$
C_{\alpha_{1} \ldots \alpha_{2 s}}^{(2)}=\sum_{i, j} a\left(m_{i}, m_{j} ; z\right) \varkappa_{\left(\alpha_{1} \alpha_{2}\right.} \ldots \varkappa_{\left.\alpha_{2 s-1} \alpha_{2 s}\right)},
$$

where $a\left(m_{i}, m_{j} ; z\right)$ are some bilinear $z$-dependent coefficients of HS parameters $m_{s}$, (4.6). Thus, the algebraic type is again Petrov $D$.

- Spin finiteness. Suppose $f(y)$ is polynomial corresponding to a finite amount of nonzero HS spins at free level, then the quadratic correction (4.53) contains no more than finite number of planar HS excitation too. For example, should one starts out with just an $s=2$ black brane at free level

$$
f_{2}(w)=\frac{m_{2}}{2}\left(\frac{z}{2} \varkappa_{\alpha \beta} w^{\alpha} w^{\beta}\right)^{2},
$$

then the quadratic correction calculated from (4.53) cuts short at $s=4$ given by the last term below

$$
\phi(w ; z)=\frac{1}{5} m_{2}^{2} z^{5}+\frac{7}{6} m_{2}^{2} z^{3}\left(\frac{z}{2} \varkappa_{\alpha \beta} w^{\alpha} w^{\beta}\right)^{2}+\frac{71}{280} m_{2}^{2} z\left(\frac{z}{2} \varkappa_{\alpha \beta} w^{\alpha} w^{\beta}\right)^{4} .
$$

This is the general feature of our solution. If $f(w)$ is bounded by some spin $s$, then the deformation terminates at $s_{\max }=2 s$. At the same time any such spin $s>0$ induces a tower of nonzero spins $0, \ldots, 2 s$.

Note that while $s=2$ Weyl tensor (4.57) is exactly the same at the linearized level as of a GR black brane, this is no longer so in HS interactions. From (4.58) it follows that not only spin $s=2$ receives correction that brings it away from ordinary black brane, but also the new fields $s=0$ and $s=4$ show up.

The corrections from the $s=2$ sector to itself are nonzero since the gravity interaction differs from that of Einstein within the HS theory. This leads to the breakdown of the planar GR black hole (4.57) already by quadratic HS corrections.

- Multi-copy structure. Since by construction solution (4.53) is a deformation of the linearized solution, which has a structure of the double copy, it enjoys the double copy or better put it the multi-copy property too. Indeed, as is seen from (4.56), each spin $s$ HS Weyl tensor is expressed via a multiple product of spin $s=1$ solution

$$
C_{\alpha \beta}=\frac{1}{2} z^{3} \varkappa_{\alpha \beta}, \quad \bar{C}_{\dot{\alpha} \dot{\beta}}=\frac{1}{2} z^{3} \bar{\varkappa}_{\dot{\alpha} \dot{\beta}},
$$


where from (3.4) $z$ itself is the zeroth copy of Maxwell field

$$
z=\left(-2 C_{\alpha \beta} C^{\alpha \beta}\right)^{\frac{1}{4}}
$$

Therefore, the spin $s$ HS Weyl tensor from (4.56) acquires a form of a power $s$ of Maxwell field (4.59) up to a $z$-dependent prefactor which can be expressed via zeroth copy (4.60). The multi-copy results from Petrov $D$-type form of the solution and its sufficiently reach global symmetry.

- Closed scalar sector. While it follows from (4.53) that every spin contribution in $f(y)$ induces lower and higher spins as is generally expected for HS algebraic reason, this is not so for $f=1$. This case corresponds to a single $\Delta=1$ scalar $C=z$. Quadratic corrections to the spin $s=0$ sector should vanish in general because scalar vertex $0-0-0$ is absent in four dimensions [51] being conformally invariant [56]. This is indeed what happens for $f=1$ as one finds from $(4.53) \phi(0 ; z)=0$. But it turns out that the result is even stronger than that. In this case we have

$$
\phi(w ; z)=0,
$$

which means that there are no corrections from $s=0$ to spins $s>0$ either. In other words, $f=1$ is an exact solution at this order. It will be interesting to see whether it remains so at higher orders.

- Higher spin scalar condensate. An interesting property of (4.53) comes from inspection of its scalar sector. Let us take a general

$$
f(w)=\sum_{s=0} \frac{m_{s}}{s !}\left(\frac{z}{2} \varkappa_{\alpha \beta} w^{\alpha} w^{\beta}\right)^{s}
$$

with a priori arbitrary HS real parameters $m_{s}$ and let us find its contribution to the scalar sector from (4.53). Straightforward calculation gives

$$
\phi(0 ; z)=\sum_{s=1} \frac{m_{s}^{2}}{2 s+1} z^{2 s+1} .
$$

Remarkably, the obtained contribution turns out to be diagonal in spins and is strictly positive for $z>0$ no matter what signs of HS charges $m_{s}$ are. So we see that HS fields at this order condensate into a positive scalar. Note also that such a correction affects neither free $\Delta=1$, nor $\Delta=2$ scalar boundary behavior. It would be very interesting to understand if there are any fundamental grounds that lead to positivity of scalar perturbation.

An example of what (4.63) could be if, lets say, all HS parameters for every integer spin are equal to each other $m_{s}=m$ is

$$
\phi(0 ; z)=m^{2}(\operatorname{arctanh} z-z)
$$

leading to the following second order corrections to the scalar field

$$
C^{(2)}(0,0 ; z)=2 m^{2} z(\operatorname{arctanh} z-z)=\frac{2}{3} m^{2} z^{4}+O\left(z^{6}\right) .
$$




\section{Concluding remarks}

In this paper we have completed our analysis of the bosonic planar HS solutions at leading interaction order. At the linearized level such solutions have been proposed in [4] while the nonlinear deformation in the sector of HS curvatures is found here.

Solving nonlinear HS equations order by order is generally a highly nontrivial task. The problem is twofold. First off, HS equations contain higher derivatives which grow with spin making HS equations quite complicated already at leading interaction order. Second, it is not infrequently that even the simplest free solutions have increasingly complicated with spin form, especially those in the sector of gauge fields (see e.g., [57, 58] for the plane wave like examples in AdS). Both problems together normally square difficulties of perturbative expansion.

What makes life easier in our case is a remarkably simple linearized version of HS planar solution (4.3), (4.4). It exists in the type- $A$ HS theory only with the phase parameter $\eta=1$ and we expect that a similar solution away from this point would be much more complicated. Moreover, being static and having planar symmetry the solution appears to be perfectly suited for what we call current ansatz (3.30), which allows one isolating HS currents from the twisted-adjoint module. In terms of 'currents' each spin planar contribution corresponds to a simple monomial. Originally introduced in [48] for HS bulk-boundary analysis at free level, the current ansatz unexpectedly goes through in interactions [49]. Having generalized this ansatz to make it covariant with respect to planar symmetry, we use it here at the nonlinear level and this has brought us extra simplification.

A great deal of technical analysis is concerned with checking whether the linearized solution admits nonlinear deformation or not. Putting it differently, the question is whether the nonlinear solution depends on the same set of fields as the free one. Practically, this amounts to verifying static constraint (4.28) for particular HS interactions. We found that it is satisfied for the standard bosonic HS model, while this may not be the case for a different type of embeddings into the susy HS system. Particular example of the so called chiral embedding (2.30) was shown not to respect the static constraint.

In the bosonic case we found quadratic corrections to HS Weyl tensors in a closed form (4.54). These turned out to be algebraically special of Petrov type $D$. Moreover, in the spirit of the Weyl double copy, the final result can be reconstructed from the free spin $s=1$ solution polynomially using the zeroth copy scalar for every spin $s$. Therefore the obtained solution naturally generalizes the double copy form of GR black holes observed in [32] (see also [35]) to nonlinear HS interacting level.

The solution is characterized by an arbitrary real function $f(y)$ or equivalently by (in)finitely many real arbitrary HS parameters $m_{s}$ that show up in its Taylor expansion and generalize the mass parameter $m_{2}$ of a black brane. In particular, the case of a finite number of $m_{s}$ corresponds to a polynomial $f(y)$. The nonlinear corrections come out as bilinears in $m_{s}$. Their properties are collected in section 4.4 .

Some notable features are as follows. While generally one may expect that a given spin $s$ induces infinitely many spins in interactions due to HS algebraic argument, in practice it did not happen as quadratic corrections terminated at spin $2 s$ for kinematical reason. 
Particularly, spin $s=0$ yields no quadratic corrections at all including to itself and thus forms a closed interacting sector. Moreover, one may argue that there would be no higher order corrections to $s>0$ from a scalar either due to kinematical reasons as well.

Another interesting feature is HS contribution into scalar sector (4.63). While as a matter of principle HS parameters can be either positive or negative, their quadratic corrections to the scalar turn out to be diagonal in $m_{s}$ and are strictly positive manifesting universal behavior for all spins. It would be very interesting to explore this phenomenon further.

Using (4.3) as a free solution we left aside $\Delta=2$ scalar (4.9). We did that purposely as on one hand the extra scalar brings in considerable complication of the analysis, yet seemingly changing no qualitative picture of interactions. But on the other, the whole scalar sector alone deserves a separate consideration. The global symmetry is bigger for pure scalar than the planar one considered in the presence of spinning fields. It is given by the Poincaré symmetry of the three dimensional boundary. Given that at the full nonlinear level a combination of $\Delta=1$ and $\Delta=2$ scalars arguably induces no fields $s>0$ for kinematical reasons, it would be very interesting to find the corresponding exact solution. If this is possible then one will be able addressing questions on HS symmetry breaking by that potentially simple yet very symmetric HS vacuum, which would be just the $A d S_{4}$ in its gravity sector. It is intriguing to obtain the mass spectrum that such a symmetry breaking may deliver ${ }^{11}$ (see [59] for a proposal in this direction and also [60] for phenomenological application of the conformal HS symmetry breaking). We hope to consider this problem elsewhere.

Another comment concerns the double copy form of the solution. Though the obtained leading order corrections appear to have the multi-copy form, it was not granted in the first place. An example of chiral embedding (2.30) that has a multi-copy structure at free level proves that it may not remain so in perturbations. Indeed since the quadratic corrections fail to respect static constaint (4.28), the solution depends on some extra fields that are not present at first order. Therefore, the corresponding HS Weyl tensors can not be expressed in terms of Maxwell tensor (4.59).

Let us conclude by what our analysis adds to lower spin interaction within HS theory. There is an interesting phenomenon of GR black holes that make them linearly exact. For that reason the $s=2$ component of the linearized solution (4.3) describes the standard black brane Weyl tensor. One could have hoped that it may not be affected by HS interactions either, but this is actually not happening. Not only $s=2$ gets corrected by second order, it inevitably induces nontrivial $s=4$ field. More generally, the lower spin system of $s=0,1,2$ that admits planar symmetry is not closed under HS interactions for any values of their charges.

\section{Acknowledgments}

We are grateful to Ruslan Metsaev for useful discussion on higher-spin symmetry breaking and to Mitya Ponomarev and M.A. Vasiliev for valuable comments on the draft of the paper. We would also like to thank Tim Adamo for correspondence. This research was supported by the Russian Science Foundation grant 18-12-00507.

\footnotetext{
${ }^{11}$ We are grateful to Ruslan Metsaev for the very useful discussion on this point.
} 


\section{A Verification of consistency condition}

In this section consistency condition (4.28) is explicitly checked for bosonic embedding (4.10). Symmetric part of the current can be treated as a sum of several contributions, namely

$$
J_{\alpha \beta}=J_{\alpha \beta}^{\omega}+\widehat{J}_{\alpha \beta}^{\eta}+\widehat{J}_{\alpha \beta}^{\bar{\eta}}+\widetilde{J}_{\alpha \beta}^{\eta}+\widetilde{J}_{\alpha \beta}^{\bar{\eta}} .
$$

We split $J_{\alpha \beta}^{c}$ into two terms compared to (4.16). First term given by (4.19) originates from commutator of the first order correction to field $\mathbf{w}$ with field $\mathcal{C}$, while all other terms come from the holomorphic and antiholomorphic vertices on AdS background. Even though our analysis requires to set phase parameter of the Vasiliev theory to one (4.2), it is useful to separate terms proportional to $\eta$ from those proportional to $\bar{\eta}$ to keep track of cancellations. Explicit expressions for all of the contributions are provided below

$$
\begin{aligned}
J_{\alpha \beta}^{\omega}= & \frac{i}{z} \int_{0}^{1} d t\left\{\int \frac{\mathrm{d} u \mathrm{~d} v}{(2 \pi)^{2}} e^{\frac{i}{z} u_{\alpha} v^{\alpha}} f(w+v) \frac{\partial^{2}}{\partial w^{\alpha} \partial w^{\beta}} f(y+\bar{y}+t u)\right. \\
& +\int \frac{\mathrm{d} \bar{u} \mathrm{~d} \bar{v}}{(2 \pi)^{2}} e^{-\frac{i}{z} \bar{u}_{\alpha} \bar{v}^{\alpha}} f(\bar{y}+\bar{v}) \frac{\partial^{2}}{\partial w^{\alpha} \partial w^{\beta}} f(y+\bar{y}+t \bar{u}) \\
& -\int \frac{\mathrm{d} u \mathrm{~d} v}{(2 \pi)^{2}} e^{\frac{i}{z} u_{\alpha} v^{\alpha}} f(y+u) \frac{\partial^{2}}{\partial w^{\alpha} \partial w^{\beta}} f(y-\bar{y}+t v) \\
& \left.-\int \frac{\mathrm{d} \bar{u} \mathrm{~d} \bar{v}}{(2 \pi)^{2}} e^{-\frac{i}{z} \bar{u}_{\alpha} \bar{v}^{\alpha}} f(\bar{y}+\bar{u}) \frac{\partial^{2}}{\partial w^{\alpha} \partial w^{\beta}} f(y-\bar{y}-t \bar{v})\right\}, \\
\widehat{J}_{\alpha \beta}^{\eta}= & -\frac{1}{2} \int_{0}^{1} d t\left\{w_{\alpha} f(t w) \frac{\partial}{\partial w^{\beta}}(f(\bar{w}-t w)+f(\bar{w}+t w))\right. \\
& \left.+w_{\beta} f(t w) \frac{\partial}{\partial w^{\alpha}}(f(\bar{w}-t w)+f(\bar{w}+t w))\right\}, \\
\widehat{J}_{\alpha \beta}^{\bar{\eta}}= & \frac{1}{2} \int_{0}^{1} d t\left\{\bar{w}_{\alpha} f(t \bar{w}) \frac{\partial}{\partial \bar{w}^{\beta}}(f(w-t \bar{w})+f(w+t \bar{w}))\right. \\
& \left.+\bar{w}_{\beta} f(t \bar{w}) \frac{\partial}{\partial \bar{w}^{\alpha}}(f(w-t \bar{w})+f(w+t \bar{w}))\right\}, \\
\widetilde{J}_{\alpha \beta}^{\eta}= & -\frac{1}{2 z^{2}}\left(w_{\alpha} \frac{\partial}{\partial w^{\beta}}+w_{\beta} \frac{\partial}{\partial w^{\alpha}}\right) \\
& \times \int_{0}^{1} d t \int \frac{\mathrm{d} \bar{u} \mathrm{~d} \bar{v}}{(2 \pi)^{2}} e^{-\frac{i}{z} \bar{u}_{\alpha} \bar{v}^{\alpha}} f(\bar{w}+(1-t) w+\bar{u}) f(\bar{w}+\bar{v}-t w), \\
& \frac{1}{2 z^{2}}\left(\bar{w}_{\alpha} \frac{\partial}{\partial \bar{w}^{\beta}}+\bar{w}_{\beta} \frac{\partial}{\partial \bar{w}^{\alpha}}\right) \\
& \times \int_{0}^{1} d t \int \frac{\mathrm{d} u \mathrm{~d} v}{(2 \pi)^{2}} e^{\frac{i}{z} u_{\alpha} v^{\alpha}} f(w+(1-t) \bar{w}+u) f(w+v-t \bar{w}) . \\
\widetilde{J}_{\alpha \beta} \bar{w}_{\alpha \beta} &
\end{aligned}
$$

To proceed with consistency check we apply derivatives to all of the contributions and contract respective indices. Using partial integration and Schouten identities, the results of differentiation of each contribution (A.2)-(A.6) are the following

$$
\begin{aligned}
\frac{\partial}{\partial w^{\beta}} J_{\alpha}^{\omega}{ }_{\alpha}^{\beta}(w, \bar{w})= & -\frac{1}{z^{2}} \int \frac{\mathrm{d} u \mathrm{~d} v}{(2 \pi)^{2}} e^{\frac{i}{z} u_{\alpha} v^{\alpha}} f(w+v) \frac{\partial}{\partial w^{\alpha}} f(w+\bar{w}+u) \\
& -\frac{1}{z^{2}} \int \frac{\mathrm{d} u \mathrm{~d} v}{(2 \pi)^{2}} e^{\frac{i}{z} u_{\alpha} v^{\alpha}} f(w+u) \frac{\partial}{\partial w^{\alpha}} f(w-\bar{w}+v) \\
& +\frac{\partial}{\partial \bar{w}^{\alpha}}(f(w)(f(w+\bar{w})-f(w-\bar{w}))),
\end{aligned}
$$




$$
\begin{aligned}
\frac{\partial}{\partial w^{\beta}} \widetilde{J}_{\alpha}^{\bar{\eta}}{ }_{\alpha}^{\beta}(w, \bar{w})= & \frac{1}{z^{2}} \frac{\partial}{\partial \bar{w}^{\alpha}}\left[\int \frac{\mathrm{d} u \mathrm{~d} v}{(2 \pi)^{2}} e^{\frac{i}{z} u_{\alpha} v^{\alpha}} f(w+u+\bar{w}) f(y+v)\right. \\
& \left.-\int \frac{\mathrm{d} u \mathrm{~d} v}{(2 \pi)^{2}} e^{\frac{i}{z} u_{\alpha} v^{\alpha}} f(w+u) f(w+v-\bar{w})\right] \\
& -\frac{1}{z^{2}} \frac{\partial}{\partial w^{\alpha}} \int_{0}^{1} d t \int \frac{\mathrm{d} u \mathrm{~d} v}{(2 \pi)^{2}} e^{\frac{i}{z} u_{\alpha} v^{\alpha}} f(w+u+(1-t) \bar{w}) f(w+v-t \bar{w}) \\
& -\frac{1}{2 z^{2}} \frac{\partial}{\partial w^{\alpha}}\left(\bar{w}^{\sigma} \frac{\partial}{\partial \bar{w}^{\sigma}}\right) \\
& \times \int_{0}^{1} d t \int \frac{\mathrm{d} u \mathrm{~d} v}{(2 \pi)^{2}} e^{\frac{i}{z} u_{\alpha} v^{\alpha}} f(w+u+(1-t) \bar{w}) f(w+v-t \bar{w}), \\
\frac{\partial}{\partial w^{\beta}} \widetilde{J}_{\alpha}^{\eta}(w, \bar{w})= & -\frac{1}{z^{2}} \frac{\partial}{\partial w^{\alpha}} \int_{0}^{1} d t \int \frac{\mathrm{d} \bar{u} \mathrm{~d} \bar{v}}{(2 \pi)^{2}} e^{-\frac{i}{z} \bar{u}_{\alpha} \bar{v}^{\alpha}} f(\bar{w}+(1-t) w+\bar{u}) f(\bar{w}+\bar{v}-t w) \\
& -\frac{1}{2 z^{2}} \frac{\partial}{\partial w^{\alpha}}\left(w^{\sigma} \frac{\partial}{\partial w^{\sigma}}\right) \\
& \times \int_{0}^{1} d t \int \frac{\mathrm{d} \bar{u} \mathrm{~d} \bar{v}}{(2 \pi)^{2}} e^{-\frac{i}{z} \bar{u}_{\alpha} \bar{v}^{\alpha}} f(\bar{w}+(1-t) w+\bar{u}) f(\bar{w}+\bar{v}-t w) . \\
\frac{\partial}{\partial w^{\beta}} \widehat{J}_{\alpha}^{\eta}{ }_{\alpha}^{\beta}(w, \bar{w})= & \frac{\partial}{2} \frac{\partial}{\partial w^{\alpha}} \int_{0}^{1} d t t\left(w^{\sigma} \frac{\partial}{\partial \bar{w}^{\sigma}}\right) f(t w)(f(\bar{w}+t w)-f(\bar{w}-t w)) \\
& -\eta \frac{\partial}{\partial \bar{w}^{\alpha}} f(w)(f(\bar{w}+w)-f(\bar{w}-w)), \\
\frac{\partial}{\partial w^{\beta}} \widehat{J}_{\alpha}^{\eta}{ }_{\alpha}^{\beta}(w, \bar{w})= & \frac{1}{2} \frac{\partial}{\partial w^{\alpha}} \int_{0}^{1} d t t\left(\bar{w}^{\sigma} \frac{\partial}{\partial w^{\sigma}}\right)[f(t \bar{w})(f(w+t \bar{w})-f(w-t \bar{w}))] .
\end{aligned}
$$

Summing up all the contributions one obtains

$$
\begin{aligned}
\partial_{\beta} J_{\alpha}^{\beta}(w, \bar{w})= & \frac{\partial}{\partial w^{\alpha}}\left[-\frac{1}{z^{2}} \int_{0}^{1} d t \int \frac{\mathrm{d} u \mathrm{~d} v}{(2 \pi)^{2}} e^{\frac{i}{z} u_{\alpha} v^{\alpha}} f(w+u+(1-t) \bar{w}) f(w+v-t \bar{w})\right. \\
& -\frac{1}{z^{2}} \int_{0}^{1} d t \int \frac{\mathrm{d} \bar{u} \mathrm{~d} \bar{v}}{(2 \pi)^{2}} e^{-\frac{i}{z} \bar{u}_{\alpha} \bar{v}^{\alpha}} f(\bar{w}+(1-t) w+\bar{u}) f(\bar{w}+\bar{v}-t w) \\
& -\frac{1}{2 z^{2}}\left(\bar{w}^{\sigma} \frac{\partial}{\partial \bar{w}^{\sigma}}\right) \int_{0}^{1} d t \int \frac{\mathrm{d} u \mathrm{~d} v}{(2 \pi)^{2}} e^{\frac{i}{z} u_{\alpha} v^{\alpha}} f(w+u+(1-t) \bar{w}) f(w+v-t \bar{w}) \\
& -\frac{1}{2 z^{2}}\left(w^{\sigma} \frac{\partial}{\partial w^{\sigma}}\right) \int_{0}^{1} d t \int \frac{\mathrm{d} \bar{u} \mathrm{~d} \bar{v}}{(2 \pi)^{2}} e^{-\frac{i}{z} \bar{u}_{\alpha} \bar{v}^{\alpha}} f(\bar{y}+(1-t) y+\bar{u}) f(\bar{y}+\bar{v}-t y) \\
& +\frac{1}{2} \int_{0}^{1} d t t\left(w^{\sigma} \frac{\partial}{\partial \bar{w}^{\sigma}}\right) f(t w)(f(\bar{w}+t w)-f(\bar{w}-t w)) \\
& \left.+\frac{1}{2} \int_{0}^{1} d t t\left(\bar{w}^{\sigma} \frac{\partial}{\partial w^{\sigma}}\right)[f(t \bar{w})(f(w+t \bar{w})-f(w-t \bar{w}))]\right] .
\end{aligned}
$$

Analogously one can compute contraction with barred derivative

$$
\begin{aligned}
\bar{\partial}_{\beta} J_{\alpha}^{\beta}(w, \bar{w})= & \frac{\partial}{\partial \bar{w}^{\alpha}}\left[\frac{1}{z^{2}} \int_{0}^{1} d t \int \frac{\mathrm{d} \bar{u} \mathrm{~d} \bar{v}}{(2 \pi)^{2}} e^{-\frac{i}{z} \bar{u}_{\alpha} \bar{v}^{\alpha}} f(\bar{w}+(1-t) w+\bar{u}) f(\bar{w}+v-t w)\right. \\
& +\frac{1}{z^{2}} \int_{0}^{1} d t \int \frac{\mathrm{d} u \mathrm{~d} v}{(2 \pi)^{2}} e^{\frac{i}{z} u_{\alpha} v^{\alpha}} f(w+u+(1-t) \bar{w}) f(w+v-t \bar{w}) \\
& +\frac{1}{2 z^{2}}\left(w^{\sigma} \frac{\partial}{\partial w^{\sigma}}\right) \int_{0}^{1} d t \int \frac{\mathrm{d} \bar{u} \mathrm{~d} \bar{v}}{(2 \pi)^{2}} e^{-\frac{i}{z} \bar{u}_{\alpha} \bar{v}^{\alpha}} f(\bar{w}+(1-t) w+\bar{u}) f(\bar{w}+\bar{v}-t w)
\end{aligned}
$$




$$
\begin{aligned}
& +\frac{1}{2 z^{2}}\left(\bar{w}^{\sigma} \frac{\partial}{\partial \bar{w}^{\sigma}}\right) \int_{0}^{1} d t \int \frac{\mathrm{d} u \mathrm{~d} v}{(2 \pi)^{2}} e^{\frac{i}{z} u_{\alpha} v^{\alpha}} f(w+u+(1-t) \bar{w}) f(w+v-t \bar{w}) \\
& -\frac{1}{2} \int_{0}^{1} d t t\left(w^{\sigma} \frac{\partial}{\partial \bar{w}^{\sigma}}\right)[f(t w)(f(\bar{w}+t w)-f(\bar{w}-t w))] \\
& \left.-\frac{1}{2} \int_{0}^{1} d t t\left(\bar{w}^{\sigma} \frac{\partial}{\partial w^{\sigma}}\right)[f(t \bar{w})(f(w+t \bar{w})-f(w-t \bar{w}))]\right] .
\end{aligned}
$$

After bringing the right hand sides of (A.12) and (A.13) to the form of total derivatives, it is clear that consistency conditions (4.27) and (4.28) are both trivially satisfied.

\section{B Explicit expression for $h(w, \bar{w})$}

The integral over $\tau$ in formula (4.36) can be computed (via partial integration). Below we provide the final result of this calculation

$$
\begin{aligned}
h(w, \bar{w})= & \int_{0}^{1} d t\left\{\frac{1}{z^{2}} \int \frac{\mathrm{d} \bar{u} \mathrm{~d} \bar{v}}{(2 \pi)^{2}} e^{-\frac{i}{z} \bar{u} \bar{v}} f(\bar{w}+(1-t) w+\bar{u}) f(\bar{w}+\bar{v}-t w)\right. \\
& +\frac{1}{z^{2}} \int \frac{\mathrm{d} u \mathrm{~d} v}{(2 \pi)^{2}} e^{\frac{i}{z} u v} f(w+u+(1-t) \bar{w}) f(y+v-t \bar{y}) \\
& +\frac{1}{2 z^{2}}\left(w^{\sigma} \frac{\partial}{\partial w^{\sigma}}\right) \int \frac{\mathrm{d} \bar{u} \mathrm{~d} \bar{v}}{(2 \pi)^{2}} e^{-\frac{i}{z} \bar{u} \bar{v}} f(\bar{w}+(1-t) w+\bar{u}) f(\bar{w}+\bar{v}-t w) \\
& +\frac{1}{2 z^{2}}\left(\bar{w}^{\sigma} \frac{\partial}{\partial \bar{w}^{\sigma}}\right) \int \frac{\mathrm{d} u \mathrm{~d} v}{(2 \pi)^{2}} e^{\frac{i}{z} u v} f(w+u+(1-t) \bar{w}) f(w+v-t \bar{w}) \\
& -\frac{2}{z^{2}} \int \frac{\mathrm{d} u \mathrm{~d} v}{(2 \pi)^{2}} e^{\frac{i}{z} u_{\alpha} v^{\alpha}} f(u) f(v) \\
& -\frac{1}{2} f(t w)\left(w^{\sigma} \frac{\partial}{\partial w^{\sigma}}\right)(f(\bar{w}+t w)+f(\bar{w}-t w)) \\
& \left.-\frac{1}{2} f(t \bar{w})\left(\bar{w}^{\sigma} \frac{\partial}{\partial \bar{w}^{\sigma}}\right)(f(w+t \bar{w})-f(w-t \bar{w}))\right\}+\mathbf{c}(z) .
\end{aligned}
$$

Open Access. This article is distributed under the terms of the Creative Commons Attribution License (CC-BY 4.0), which permits any use, distribution and reproduction in any medium, provided the original author(s) and source are credited.

\section{References}

[1] J.M. Maldacena, The large $N$ limit of superconformal field theories and supergravity, Int. J. Theor. Phys. 38 (1999) 1113 [Adv. Theor. Math. Phys. 2 (1998) 231] [hep-th/9711200] [INSPIRE].

[2] S.S. Gubser, I.R. Klebanov and A.M. Polyakov, Gauge theory correlators from noncritical string theory, Phys. Lett. B 428 (1998) 105 [hep-th/9802109] [INSPIRE].

[3] E. Witten, Anti-de Sitter space and holography, Adv. Theor. Math. Phys. 2 (1998) 253 [hep-th/9802150] [INSPIRE].

[4] V.E. Didenko and A.V. Korybut, Planar solutions of higher-spin theory. Part I. Free field level, JHEP 08 (2021) 144 [arXiv:2105.09021] [INSPIRE]. 
[5] D. Birmingham, Topological black holes in Anti-de Sitter space, Class. Quant. Grav. 16 (1999) 1197 [hep-th/9808032] [INSPIRE].

[6] M.A. Vasiliev, Higher spin gauge theories: star product and AdS space, hep-th/9910096 [INSPIRE].

[7] M.A. Vasiliev, More on equations of motion for interacting massless fields of all spins in (3+1)-dimensions, Phys. Lett. B 285 (1992) 225 [inSPIRE].

[8] O.A. Gelfond and M.A. Vasiliev, Homotopy operators and locality theorems in higher-spin equations, Phys. Lett. B $\mathbf{7 8 6}$ (2018) 180 [arXiv:1805.11941] [INSPIRE].

[9] V.E. Didenko, O.A. Gelfond, A.V. Korybut and M.A. Vasiliev, Homotopy properties and lower-order vertices in higher-spin equations, J. Phys. A 51 (2018) 465202 [arXiv: 1807.00001] [INSPIRE].

[10] O. Aharony, S.M. Chester and E.Y. Urbach, A derivation of AdS/CFT for vector models, JHEP 03 (2021) 208 [arXiv:2011.06328] [INSPIRE].

[11] S.R. Das and A. Jevicki, Large $N$ collective fields and holography, Phys. Rev. D 68 (2003) 044011 [hep-th/0304093] [INSPIRE].

[12] A. Jevicki and J. Yoon, Bulk from bi-locals in thermo field CFT, JHEP 02 (2016) 090 [arXiv: 1503.08484] [INSPIRE].

[13] S.F. Prokushkin and M.A. Vasiliev, Higher spin gauge interactions for massive matter fields in 3D AdS space-time, Nucl. Phys. B 545 (1999) 385 [hep-th/9806236] [INSPIRE].

[14] E. Sezgin and P. Sundell, An exact solution of $4 D$ higher-spin gauge theory, Nucl. Phys. B 762 (2007) 1 [hep-th/0508158] [INSPIRE].

[15] C. Iazeolla, E. Sezgin and P. Sundell, Real forms of complex higher spin field equations and new exact solutions, Nucl. Phys. B 791 (2008) 231 [arXiv:0706.2983] [INSPIRE].

[16] V.E. Didenko and M.A. Vasiliev, Static BPS black hole in 4d higher-spin gauge theory, Phys. Lett. B 682 (2009) 305 [Erratum ibid. 722 (2013) 389] [arXiv:0906.3898] [INSPIRE].

[17] C. Iazeolla and P. Sundell, Families of exact solutions to Vasiliev's 4D equations with spherical, cylindrical and biaxial symmetry, JHEP 12 (2011) 084 [arXiv:1107.1217] [INSPIRE].

[18] J. Bourdier and N. Drukker, On classical solutions of 4d supersymmetric higher spin theory, JHEP 04 (2015) 097 [arXiv: 1411.7037] [INSPIRE].

[19] C. Iazeolla and J. Raeymaekers, On big crunch solutions in Prokushkin-Vasiliev theory, JHEP 01 (2016) 177 [arXiv: 1510.08835] [INSPIRE].

[20] P. Sundell and Y. Yin, New classes of bi-axially symmetric solutions to four-dimensional Vasiliev higher spin gravity, JHEP 01 (2017) 043 [arXiv:1610.03449] [INSPIRE].

[21] C. Iazeolla and P. Sundell, 4D higher spin black holes with nonlinear scalar fluctuations, JHEP 10 (2017) 130 [arXiv: 1705.06713] [INSPIRE].

[22] C. Iazeolla, E. Sezgin and P. Sundell, On exact solutions and perturbative schemes in higher spin theory, Universe 4 (2018) 5 [arXiv:1711.03550] [INSPIRE].

[23] R. Aros, C. Iazeolla, J. Noreña, E. Sezgin, P. Sundell and Y. Yin, FRW and domain walls in higher spin gravity, JHEP 03 (2018) 153 [arXiv: 1712.02401] [INSPIRE]. 
[24] X. Bekaert, J. Erdmenger, D. Ponomarev and C. Sleight, Quartic AdS interactions in higher-spin gravity from conformal field theory, JHEP 11 (2015) 149 [arXiv:1508.04292] [INSPIRE].

[25] C. Sleight and M. Taronna, Higher-spin gauge theories and bulk locality, Phys. Rev. Lett. 121 (2018) 171604 [arXiv:1704.07859] [INSPIRE].

[26] M.A. Vasiliev, Current interactions and holography from the 0-form sector of nonlinear higher-spin equations, JHEP 10 (2017) 111 [arXiv:1605.02662] [INSPIRE].

[27] V.E. Didenko, O.A. Gelfond, A.V. Korybut and M.A. Vasiliev, Limiting shifted homotopy in higher-spin theory and spin-locality, JHEP 12 (2019) 086 [arXiv:1909.04876] [INSPIRE].

[28] O.A. Gelfond and M.A. Vasiliev, Spin-locality of higher-spin theories and star-product functional classes, JHEP 03 (2020) 002 [arXiv: 1910.00487] [INSPIRE].

[29] V.E. Didenko, O.A. Gelfond, A.V. Korybut and M.A. Vasiliev, Spin-locality of $\eta^{2}$ and $\bar{\eta}^{2}$ quartic higher-spin vertices, JHEP 12 (2020) 184 [arXiv: 2009.02811] [INSPIRE].

[30] A. David and Y. Neiman, Higher-spin symmetry vs. boundary locality, and a rehabilitation of dS/CFT, JHEP 10 (2020) 127 [arXiv:2006.15813] [inSPIRE].

[31] O.A. Gelfond and A.V. Korybut, Manifest form of the spin-local higher-spin vertex $\Upsilon_{\omega C C C}^{\eta \eta}$, Eur. Phys. J. C 81 (2021) 605 [arXiv:2101.01683] [INSPIRE].

[32] V.E. Didenko, A.S. Matveev and M.A. Vasiliev, Unfolded description of AdS $S_{4}$ Kerr black hole, Phys. Lett. B 665 (2008) 284 [arXiv:0801.2213] [INSPIRE].

[33] V.E. Didenko, A.S. Matveev and M.A. Vasiliev, Unfolded dynamics and parameter flow of generic $A d S_{4}$ black hole, arXiv:0901.2172 [INSPIRE].

[34] V.E. Didenko, Coordinate independent approach to 5d black holes, Class. Quant. Grav. 29 (2012) 025009 [arXiv: 1108.4321] [INSPIRE].

[35] R. Monteiro, D. O'Connell and C.D. White, Black holes and the double copy, JHEP 12 (2014) 056 [arXiv: 1410.0239] [INSPIRE].

[36] A. Luna, R. Monteiro, I. Nicholson and D. O'Connell, Type D spacetimes and the Weyl double copy, Class. Quant. Grav. 36 (2019) 065003 [arXiv:1810.08183] [InSPIRE].

[37] H. Kawai, D.C. Lewellen and S.H.H. Tye, A relation between tree amplitudes of closed and open strings, Nucl. Phys. B 269 (1986) 1 [INSPIRE].

[38] T. Adamo and A. Ilderton, Classical and quantum double copy of back-reaction, JHEP 09 (2020) 200 [arXiv : 2005.05807] [INSPIRE].

[39] H. Godazgar, M. Godazgar, R. Monteiro, D. Peinador Veiga and C.N. Pope, Weyl double copy for gravitational waves, Phys. Rev. Lett. 126 (2021) 101103 [arXiv:2010.02925] [INSPIRE].

[40] P. Ferrero and D. Francia, On the Lagrangian formulation of the double copy to cubic order, JHEP 02 (2021) 213 [arXiv: 2012.00713] [INSPIRE].

[41] C.D. White, Twistorial foundation for the classical double copy, Phys. Rev. Lett. 126 (2021) 061602 [arXiv: 2012.02479] [inSPIRE].

[42] L. Borsten, H. Kim, B. Jurčo, T. Macrelli, C. Sämann and M. Wolf, Double copy from homotopy algebras, Fortsch. Phys. 69 (2021) 2100075 [arXiv:2102.11390] [INSPIRE]. 
[43] E. Chacón, S. Nagy and C.D. White, The Weyl double copy from twistor space, JHEP 05 (2021) 2239 [arXiv:2103.16441] [INSPIRE].

[44] X. Zhou, Double copy relation in AdS space, Phys. Rev. Lett. 127 (2021) 141601 [arXiv:2106.07651] [INSPIRE].

[45] E. Chacón, A. Luna and C.D. White, The double copy of the multipole expansion, arXiv:2108.07702 [INSPIRE].

[46] H. Godazgar, M. Godazgar, R. Monteiro, D. Peinador Veiga and C.N. Pope, Asymptotic Weyl double copy, JHEP 11 (2021) 126 [arXiv:2109.07866] [INSPIRE].

[47] T. Adamo and U. Kol, Classical double copy at null infinity, arXiv:2109.07832 [INSPIRE].

[48] M.A. Vasiliev, Holography, unfolding and higher-spin theory, J. Phys. A 46 (2013) 214013 [arXiv: 1203.5554] [INSPIRE].

[49] V.E. Didenko and M.A. Vasiliev, Test of the local form of higher-spin equations via AdS/CFT, Phys. Lett. B 775 (2017) 352 [arXiv:1705.03440] [InSPIRE].

[50] M.A. Vasiliev, Consistent equations for interacting massless fields of all spins in the first order in curvatures, Annals Phys. 190 (1989) 59.

[51] E. Sezgin and P. Sundell, Holography in $4 D$ (super) higher spin theories and a test via cubic scalar couplings, JHEP 07 (2005) 044 [hep-th/0305040] [INSPIRE].

[52] V.E. Didenko, N.G. Misuna and M.A. Vasiliev, Charges in nonlinear higher-spin theory, JHEP 03 (2017) 164 [arXiv:1512.07626] [INSPIRE].

[53] C. Sleight and M. Taronna, Higher spin interactions from conformal field theory: the complete cubic couplings, Phys. Rev. Lett. 116 (2016) 181602 [arXiv:1603.00022] [INSPIRE].

[54] S. Giombi and X. Yin, Higher spin gauge theory and holography: the three-point functions, JHEP 09 (2010) 115 [arXiv: 0912.3462] [INSPIRE].

[55] V.E. Didenko and E.D. Skvortsov, Towards higher-spin holography in ambient space of any dimension, J. Phys. A 46 (2013) 214010 [arXiv:1207.6786] [InSPIRE].

[56] O.A. Gelfond and M.A. Vasiliev, Symmetries of higher-spin current interactions in four dimensions, Theor. Math. Phys. 187 (2016) 797 [arXiv:1510.03488] [INSPIRE].

[57] K.I. Bolotin and M.A. Vasiliev, Star product and massless free field dynamics in AdS4, Phys. Lett. B 479 (2000) 421 [hep-th/0001031] [INSPIRE].

[58] B. Nagaraj and D. Ponomarev, Spinor-helicity formalism for massless fields in AdS . Part II. Potentials, JHEP 06 (2020) 068 [arXiv:1912.07494] [InSPIRE].

[59] R.R. Metsaev, IIB supergravity and various aspects of light cone formalism in AdS space-time, hep-th/0002008 [INSPIRE].

[60] A.O. Barvinsky, CFT driven cosmology and conformal higher spin fields, Phys. Rev. D 93 (2016) 103530 [arXiv:1511.07625] [INSPIRE]. 